\title{
Comprehensive in silico Analysis of IKBKAP gene that could potentially cause Familial dysautonomia
}

Mujahed I. Mustafa ${ }^{1,2^{*}}$, Enas A. Osman ${ }^{2}$, Abdelrahman H. Abdelmoneiom², Dania M. Hassn ${ }^{2}$, Hadeel M. Yousif ${ }^{2}$, Inshrah K. Mahgoub ${ }^{2}$, Razan M. Badawi ${ }^{2}$,Kutuf A. Albushra ${ }^{2}$, Tebyan A Abdelhameed $^{2}$,Mohamed A. Hassan ${ }^{2}$

1-Department of Biochemistry, University of Bahri, Sudan

2-Department of Biotechnology, Africa city of Technology, Sudan

*Crossponding author: Mujahed I. Mustafa: email: mujahedimustafa@gmail.com

\begin{abstract}
:
Background: Familial dysautonomia (FD) is a rare neurodevelopmental genetic disorder within the larger classification of hereditary sensory and autonomic neuropathies. We aimed to identify the pathogenic SNPS in IKBKAP gene by computational analysis software's, and to determine the structure, function and regulation of their respective proteins. Materials and Methods: We carried out in silico analysis of structural effect of each SNP using different bioinformatics tools to predict SNPs influence on protein structure and function. Result: 41 novel mutations out of 973 nsSNPs that are found be deleterious effect on the IKBKAP structure and function. Conclusion: This is the first in silico analysis in IKBKAP gene to prioritize SNPS for further genetic studies.
\end{abstract}

Keywords: Familial dysautonomia (FD), neurodevelopmental genetic disorder, autonomic neuropathies, SNPs, computational analysis, diagnostic markers. 


\section{Introduction:}

Familial dysautonomia (FD; also known as "Riley-Day syndrome") is a rare neurodevelopmental genetic disorder within the larger classification of hereditary sensory and autonomic neuropathies, ${ }^{(1,2)(3)(4)(5)}$ each caused by a different genetic error associated with an increased risk for sudden death. ${ }^{(6,7)(8)}$ it is also called hereditary sensory and autonomic neuropathy type III (HSAN III) which affects $1 / 3600$ live births in the Ashkenazi Jewish population. ${ }^{(9)}$

The IKBKAP gene give specific instructions for making a protein called elongator complex protein-1 (ELP1). This protein is found in a variety of cells, including brain cells. It is part of a sixprotein complex called the elongator complex. The elongator complex plays a key role in transcription. ${ }^{(10,11)}$ The disease is caused by mutation in the IKBKAP $(E L P 1)$ gene ${ }^{(8,12)}$ that affects the splicing of the elongator-1 protein (ELP-1) (also known as IKAP). But how it functions in disease-vulnerable neurons is still unknown. The mutation weakens the 5 ' splice site of exon 20. Variable skipping of exon 20 leads to a tissue-specific reduction in the level of ELP1 protein. Which has been mapped to a $0.5-\mathrm{cM}$ region on chromosome $9 q 31$, has eluded identification. ${ }^{(13 \text {, }}$ 14)

The most common mutation, which is present on $99.5 \%$ of all FD chromosomes, is an intronic splice site mutation that results in tissue-specific skipping of exon 20 . The second FD mutation, a missense change in exon 19 (R696P), is a missense mutation that has been identified in 4 unrelated patients heterozygous for the major splice mutation. Interestingly, despite the fact that FD is a recessive disease, normal mRNA and protein are expressed in patient cells, the diagnosis of FD has been limited to individuals of Ashkenazi Jewish ${ }^{(5)}$ descent and identification of the gene has led to widespread diagnostic and carrier testing in this population. The first non-Jewish IKBKAP mutation was reported, a proline to leucine missense mutation in exon 26, (P914L). This mutation is of particular significance because it was identified in a patient who lacks one of the cardinal diagnostic criteria for the disease-pure Ashkenazi Jewish ancestry. ${ }^{(15,16)}$

The disorder disturbs cells in the autonomic nervous system, which controls involuntary actions such as digestion, breathing (patients develop neurogenic dysphagia with frequent aspiration, chronic lung disease, and chemoreflex failure leading to severe sleep disordered breathing. ${ }^{(17)}$,production of tears, and the regulation of blood pressure and body temperature. It also affects the sensory nervous system, which controls activities related to the senses. ${ }^{(4)}$ (8) (18) (19) Progressive ataxic gait is also a common symptom in FD patients. At least $50 \%$ of adults with FD require assistance with walking. ${ }^{(20)}$

The aim of this study was to identify the pathogenic SNPs in IKBKAP using in silico prediction softwares, and to determine the structure, function and regulation of their respective proteins. 
This is the first in silico analysis in IKBKAP gene to prioritize SNPs for further genetic mapping studies. The usage of in silico approach has strong impact on the identification of candidate SNPs since they are easy and less costly, and can facilitate future genetic studies. ${ }^{(21)}$

\section{Method:}

\subsection{Data mining:}

The data on human IKBKAP gene was collected from National Center for Biological Information (NCBI) web site ${ }^{(22)}$ The SNP information (protein accession number and SNP ID) of the MEFV gene was retrieved from the NCBI dbSNP (http://www.ncbi.nlm.nih.gov/snp/) and the protein sequence was collected from Swiss Prot databases (http://expasy.org/). ${ }^{(23)}$

\subsection{SIFT:}

SIFT is a sequence homology-based tool ${ }^{(24)}$ that sorts intolerant from tolerant amino acid substitutions and predicts whether an amino acid substitution in a protein will have a phenotypic Effect. Considers the position at which the change occurred and the type of amino acid change. Given a protein sequence, SIFT chooses related proteins and obtains an alignment of these proteins with the query. Based on the amino acids appearing at each position in the alignment, SIFT calculates the probability that an amino acid at a position is tolerated conditional on the most frequent amino acid being tolerated. If this normalized value is less than a cutoff, the substitution is predicted to be deleterious. SIFT scores $<0.05$ are predicted by the algorithm to be intolerant or deleterious amino acid substitutions, whereas scores $>0.05$ are considered tolerant. It is available at (http://sift.bii.a-star.edu.sg/).

\subsection{Polyphen-2:}

It is a software tool ${ }^{(25)}$ to predict possible impact of an amino acid substitution on both structure and function of a human protein by analysis of multiple sequence alignment and protein 3D structure, in addition it calculates position-specific independent count scores (PSIC) for each of two variants, and then calculates the PSIC scores difference between two variants. The higher a PSIC score difference, the higher the functional impact a particular amino acid substitution is likely to have. Prediction outcomes could be classified as probably damaging, possibly damaging or benign according to the value of PSIC as it ranges from (0_1); values closer to zero considered benign while values closer to 1 considered probably damaging and also it can be indicated by a vertical black marker inside a color gradient bar, where green is benign and red is damaging. nsSNPs that predicted to be intolerant by Sift has been submitted to Polyphen as protein sequence in FASTA format that obtained from UniproktB /Expasy after submitting the relevant ensemble protein (ESNP) there, and then we entered position of mutation, native amino acid and the new substituent for both structural and functional 
predictions. PolyPhen version

2.2 .2

is

available

at

http://genetics.bwh.harvard.edu/pph2/index.shtml

\subsection{Provean:}

Provean is a software tool ${ }^{(26)}$ which predicts whether an amino acid substitution or indel has an impact on the biological function of a protein. It is useful for filtering sequence variants to identify nonsynonymous or indel variants that are predicted to be functionally important. It is available at (https://rostlab.org/services/snap2web/).

\subsection{SNAP2:}

Functional effects of mutations are predicted with SNAP2 ${ }^{(27)}$ SNAP2 is a trained classifier that is based on a machine learning device called "neural network". It distinguishes between effect and neutral variants/non-synonymous SNPs by taking a variety of sequence and variant features into account. The most important input signal for the prediction is the evolutionary information taken from an automatically generated multiple sequence alignment. Also structural features such as predicted secondary structure and solvent accessibility are considered. If available also annotation (i.e. known functional residues, pattern, regions) of the sequence or close homologs are pulled in. In a cross-validation over 100,000 experimentally annotated variants, SNAP2 reached sustained two-state accuracy (effect/neutral) of $82 \%$ (at an AUC of 0.9). In our hands this constitutes an important and significant improvement over other methods. It is available at (https://rostlab.org/services/snap2web/ ).

\subsection{PHD-SNP:}

An online Support Vector Machine (SVM) based classifier, is optimized to predict if a given single point protein mutation can be classified as disease-related or as a neutral polymorphism, it is available at: (http://http://snps.biofold.org/phd-snp/phdsnp.html)

\subsection{SNP\& Go:}

SNPS\&GO is an algorithm developed in the Laboratory of Biocomputing at the University of Bologna directed by Prof. Rita Casadio. SNPs\&GO is an accurate method that, starting from a protein sequence, can predict whether a variation is disease related or not by exploiting the corresponding protein functional annotation. SNPS\&GO collects in unique framework information derived from protein sequence, evolutionary information, and function as encoded in the Gene Ontology terms, and outperforms other available predictive methods ${ }^{(28)}$ It is available at (http://snps.biofold.org/snps-and-go/snps-and-go.html ).

\subsection{P-Mut:}


PMUT a web-based tool (29) for the annotation of pathological variants on proteins, allows the fast and accurate prediction (approximately $80 \%$ success rate in humans) of the pathological character of single point amino acidic mutations based on the use of neural networks. It is available at (http://mmb.irbbarcelona.org/PMut ).

\subsection{I-Mutant 3.0:}

I-Mutant 3.0 Is a neural network based tool ${ }^{(30)}$ for the routine analysis of protein stability and alterations by taking into account the single-site mutations. The FASTA sequence of protein retrieved from UniProt is used as an input to predict the mutational effect on protein stability. It is available at (http://gpcr2.biocomp.unibo.it/cgi/predictors/I-Mutant3.0/I-Mutant3.0.cgi ).

\subsection{Modeling nsSNP locations on protein structure:}

Project hope (version 1.1.1) is a new online web-server to search protein 3D structures (if available) by collecting structural information from a series of sources, including calculations on the 3D coordinates of the protein, sequence annotations from the UniProt database, and predictions by DAS services. Protein sequences were submitted to project hope server in order to analyze the structural and conformational variations that have resulted from single amino acid substitution corresponding to single nucleotide substitution; It is available at (http://www.cmbi.ru.nl/hope)

\subsection{GeneMANIA:}

We submitted genes and selected from a list of data sets that they wish to query. GeneMANIA approach to know protein function prediction integrate multiple genomics and proteomics data sources to make inferences about the function of unknown proteins. (31) . It is available at (http://www.genemania.org/).

\subsection{RaptorX:}

is a web server predicting structure property of a protein sequence without using any templates. It outperforms other servers, especially for proteins without close homologs in PDB or with very sparse sequence profile. The server predicts tertiary structure. It is available at (http://raptorx.uchicago.edu/) (32) 


\section{Result:}

Table (1): Damaging or Deleterious or effect nsSNPs associated variations predicted by various softwares:

\begin{tabular}{|c|c|c|c|c|c|c|c|c|c|}
\hline \multirow[t]{2}{*}{ dbSNP rs\# } & \multirow{2}{*}{$\begin{array}{l}\text { Amino } \\
\text { Acid } \\
\text { Change }\end{array}$} & \multicolumn{2}{|l|}{ SIFT } & \multicolumn{2}{|c|}{ Polyphen } & \multicolumn{2}{|c|}{ PROVEAN } & \multicolumn{2}{|c|}{ SNAP2 } \\
\hline & & Prediction & Score & Prediction & Score & $\begin{array}{l}\text { Prediction } \\
\text { (cutoff }=-(2.5)\end{array}$ & score & Prediction & Score \\
\hline rs377679012 & R1137C & Deleterious & 0 & $\begin{array}{l}\text { PROBABLY } \\
\text { DAMAGING }\end{array}$ & 1 & Deleterious & $\begin{array}{l}- \\
7.528 \\
\end{array}$ & Effect & 63 \\
\hline rs372723869 & Y992C & Deleterious & 0 & $\begin{array}{l}\text { PROBABLY } \\
\text { DAMAGING }\end{array}$ & 1 & Deleterious & -8.41 & Effect & 58 \\
\hline rs924119518 & Y943C & Deleterious & 0 & $\begin{array}{l}\text { PROBABLY } \\
\text { DAMAGING }\end{array}$ & 1 & Deleterious & $\begin{array}{l}- \\
7.683\end{array}$ & Effect & 38 \\
\hline rs1319436059 & Y943N & Deleterious & 0 & $\begin{array}{l}\text { PROBABLY } \\
\text { DAMAGING }\end{array}$ & 1 & Deleterious & $\begin{array}{l}- \\
7.883 \\
\end{array}$ & Effect & 71 \\
\hline rs199617267 & I936T & Deleterious & 0 & $\begin{array}{l}\text { PROBABLY } \\
\text { DAMAGING }\end{array}$ & 1 & Deleterious & $\begin{array}{l}- \\
4.917 \\
\end{array}$ & Effect & 36 \\
\hline rs1218321803 & A907T & Deleterious & 0 & $\begin{array}{l}\text { PROBABLY } \\
\text { DAMAGING }\end{array}$ & 1 & Deleterious & $\begin{array}{l}- \\
3.907 \\
\end{array}$ & Effect & 32 \\
\hline rs199679232 & Y898C & Deleterious & 0 & $\begin{array}{l}\text { PROBABLY } \\
\text { DAMAGING }\end{array}$ & 1 & Deleterious & -8.79 & Effect & 30 \\
\hline rs752349406 & N888Y & Deleterious & 0 & $\begin{array}{l}\text { PROBABLY } \\
\text { DAMAGING }\end{array}$ & 1 & Deleterious & -6.68 & Effect & 36 \\
\hline rs1270126477 & D886V & Deleterious & 0 & $\begin{array}{l}\text { PROBABLY } \\
\text { DAMAGING }\end{array}$ & 1 & Deleterious & $\begin{array}{l}- \\
7.023 \\
\end{array}$ & Effect & 58 \\
\hline rs1181862976 & Y797C & Deleterious & 0 & $\begin{array}{l}\text { PROBABLY } \\
\text { DAMAGING }\end{array}$ & 1 & Deleterious & -7.59 & Effect & 75 \\
\hline rs201596987 & T793R & Deleterious & 0 & $\begin{array}{l}\text { PROBABLY } \\
\text { DAMAGING }\end{array}$ & 1 & Deleterious & $\begin{array}{l}- \\
5.089 \\
\end{array}$ & Effect & 74 \\
\hline - & D791V & Deleterious & 0 & $\begin{array}{l}\text { PROBABLY } \\
\text { DAMAGING }\end{array}$ & 1 & Deleterious & $\begin{array}{l}- \\
7.617\end{array}$ & Effect & 38 \\
\hline rs1452809051 & R749K & Deleterious & 0 & $\begin{array}{l}\text { PROBABLY } \\
\text { DAMAGING }\end{array}$ & 1 & Deleterious & $-\overline{2.928}$ & Effect & 36 \\
\hline rs370575901 & $\mathrm{R} 722 \mathrm{Q}$ & Deleterious & 0 & $\begin{array}{l}\text { PROBABLY } \\
\text { DAMAGING }\end{array}$ & 1 & Deleterious & $\begin{array}{l}- \\
3.704\end{array}$ & Effect & 79 \\
\hline rs767003999 & L716S & Deleterious & 0 & $\begin{array}{l}\text { PROBABLY } \\
\text { DAMAGING }\end{array}$ & 1 & Deleterious & $\begin{array}{l}- \\
5.572 \\
\end{array}$ & Effect & 50 \\
\hline rs1275065654 & Q710R & Deleterious & 0 & $\begin{array}{l}\text { PROBABLY } \\
\text { DAMAGING }\end{array}$ & 1 & Deleterious & $\begin{array}{l}- \\
3.937 \\
\end{array}$ & Effect & 49 \\
\hline rs763692410 & R693W & Deleterious & 0 & $\begin{array}{l}\text { PROBABLY } \\
\text { DAMAGING }\end{array}$ & 1 & Deleterious & $\begin{array}{l}- \\
7.225 \\
\end{array}$ & Effect & 88 \\
\hline rs201742754 & R689Q & Deleterious & 0 & $\begin{array}{l}\text { PROBABLY } \\
\text { DAMAGING }\end{array}$ & 1 & Deleterious & $\begin{array}{l}- \\
3.737 \\
\end{array}$ & Effect & 66 \\
\hline rs201390288 & R689W & Deleterious & 0 & $\begin{array}{l}\text { PROBABLY } \\
\text { DAMAGING }\end{array}$ & 1 & Deleterious & $\begin{array}{l} \\
7.441\end{array}$ & Effect & 80 \\
\hline rs56229130 & G576D & Deleterious & 0 & $\begin{array}{l}\text { PROBABLY } \\
\text { DAMAGING }\end{array}$ & 1 & deleterious & $\begin{array}{l}- \\
4.996\end{array}$ & Effect & 58 \\
\hline rs375957332 & P410L & Deleterious & 0 & $\begin{array}{l}\text { PROBABLY } \\
\text { DAMAGING }\end{array}$ & 1 & Deleterious & $\begin{array}{l}- \\
9.832\end{array}$ & Effect & 69 \\
\hline
\end{tabular}


bioRxiv preprint doi: https://doi.org/10.1101/436071; this version posted October 5, 2018. The copyright holder for this preprint (which was not certified by peer review) is the author/funder, who has granted bioRxiv a license to display the preprint in perpetuity. It is made available under aCC-BY-NC-ND 4.0 International license.

\begin{tabular}{|c|c|c|c|c|c|c|c|c|c|}
\hline rs1414061926 & G396V & Deleterious & 0 & $\begin{array}{l}\text { PROBABLY } \\
\text { DAMAGING }\end{array}$ & 1 & Deleterious & 8.661 & Effect & 52 \\
\hline rs775321513 & L336S & Deleterious & 0 & $\begin{array}{l}\text { PROBABLY } \\
\text { DAMAGING }\end{array}$ & 1 & Deleterious & $-\overline{4.703}$ & Effect & 38 \\
\hline rs764033545 & H329Q & Deleterious & 0 & $\begin{array}{l}\text { PROBABLY } \\
\text { DAMAGING }\end{array}$ & 1 & Deleterious & $\begin{array}{l}- \\
7.371\end{array}$ & Effect & 15 \\
\hline rs750663365 & Y328S & Deleterious & 0 & $\begin{array}{l}\text { PROBABLY } \\
\text { DAMAGING }\end{array}$ & 1 & Deleterious & $\begin{array}{l}- \\
7.288\end{array}$ & Effect & 72 \\
\hline- & $\mathrm{Y} 328 \mathrm{C}$ & Deleterious & 0 & $\begin{array}{l}\text { PROBABLY } \\
\text { DAMAGING }\end{array}$ & 1 & Deleterious & $\begin{array}{l}- \\
6.888\end{array}$ & Effect & 53 \\
\hline - & Y328F & Deleterious & 0 & $\begin{array}{l}\text { PROBABLY } \\
\text { DAMAGING }\end{array}$ & 1 & Deleterious & $\begin{array}{l}- \\
3.402\end{array}$ & Effect & 59 \\
\hline rs1159235595 & W323R & Deleterious & 0 & $\begin{array}{l}\text { PROBABLY } \\
\text { DAMAGING }\end{array}$ & 1 & Deleterious & $\begin{array}{l}- \\
12.97\end{array}$ & Effect & 89 \\
\hline rs1288706972 & S298F & Deleterious & 0 & $\begin{array}{l}\text { PROBABLY } \\
\text { DAMAGING }\end{array}$ & 1 & Deleterious & - & Effect & 72 \\
\hline rs1339594475 & G272R & Deleterious & 0 & $\begin{array}{l}\text { PROBABLY } \\
\text { DAMAGING }\end{array}$ & 1 & Deleterious & $\begin{array}{l}- \\
7.604\end{array}$ & Effect & 77 \\
\hline rs1394468770 & P248L & Deleterious & 0 & $\begin{array}{l}\text { PROBABLY } \\
\text { DAMAGING }\end{array}$ & 1 & Deleterious & $\begin{array}{l}- \\
9.498\end{array}$ & Effect & 74 \\
\hline rs1312638005 & V223E & Deleterious & 0 & $\begin{array}{l}\text { PROBABLY } \\
\text { DAMAGING }\end{array}$ & 1 & Deleterious & $\begin{array}{l}- \\
5.263\end{array}$ & Effect & 76 \\
\hline rs749382362 & R219Q & Deleterious & 0 & $\begin{array}{l}\text { PROBABLY } \\
\text { DAMAGING }\end{array}$ & 1 & Deleterious & $\begin{array}{l}- \\
3.309\end{array}$ & Effect & 58 \\
\hline rs374238430 & R219W & Deleterious & 0 & $\begin{array}{l}\text { PROBABLY } \\
\text { DAMAGING }\end{array}$ & 1 & Deleterious & $\begin{array}{l}- \\
6.451\end{array}$ & Effect & 65 \\
\hline rs1039834492 & V209E & Deleterious & 0 & $\begin{array}{l}\text { PROBABLY } \\
\text { DAMAGING }\end{array}$ & 1 & Deleterious & $-\overline{4.896}$ & Effect & 46 \\
\hline rs747349523 & D203Y & Deleterious & 0 & $\begin{array}{l}\text { PROBABLY } \\
\text { DAMAGING }\end{array}$ & 1 & Deleterious & $\begin{array}{l}- \\
8.128\end{array}$ & Effect & 84 \\
\hline rs776300430 & G202E & Deleterious & 0 & $\begin{array}{l}\text { PROBABLY } \\
\text { DAMAGING }\end{array}$ & 1 & Deleterious & - & Effect & 74 \\
\hline rs367552387 & R201Q & Deleterious & 0 & $\begin{array}{l}\text { PROBABLY } \\
\text { DAMAGING }\end{array}$ & 1 & Deleterious & $\begin{array}{l}- \\
3.409\end{array}$ & Effect & 48 \\
\hline- & R201P & Deleterious & 0 & $\begin{array}{l}\text { PROBABLY } \\
\text { DAMAGING }\end{array}$ & 1 & Deleterious & $\begin{array}{l}- \\
6.007\end{array}$ & Effect & 93 \\
\hline rs773117166 & R201W & Deleterious & 0 & $\begin{array}{l}\text { PROBABLY } \\
\text { DAMAGING }\end{array}$ & 1 & Deleterious & $\begin{array}{l}- \\
6.884\end{array}$ & Effect & 91 \\
\hline rs565257577 & G162R & Deleterious & 0 & $\begin{array}{l}\text { PROBABLY } \\
\text { DAMAGING }\end{array}$ & 1 & Deleterious & $\begin{array}{l}- \\
6.117\end{array}$ & Effect & 84 \\
\hline
\end{tabular}


Table (2): Disease effect nsSNPs associated variations predicted by various softwares:

\begin{tabular}{|c|c|c|c|c|c|c|c|c|c|}
\hline \multirow{2}{*}{ dbSNP rs\# } & \multirow{2}{*}{$\begin{array}{l}\text { Amino } \\
\text { acid } \\
\text { change }\end{array}$} & \multicolumn{3}{|c|}{ SNP\&GO } & \multicolumn{3}{|c|}{ PHD-SNP } & \multicolumn{2}{|c|}{ P-Mut } \\
\hline & & Prediction & R I & Score & Prediction & R I & Score & Score & Prediction \\
\hline rs377679012 & R1137C & Disease & 5 & 0.736 & Disease & 6 & 0.805 & $\begin{array}{c}0.90 \\
(93 \%)\end{array}$ & Disease \\
\hline rs372723869 & Y992C & Disease & 2 & 0.579 & Disease & 3 & 0.665 & $\begin{array}{c}0.54 \\
(80 \%) \\
\end{array}$ & Disease \\
\hline rs924119518 & Y943C & Disease & 0 & 0.525 & Disease & 3 & 0.629 & $\begin{array}{c}0.79 \\
(89 \%)\end{array}$ & Disease \\
\hline $\begin{array}{l}\text { rs131943605 } \\
9\end{array}$ & Y943N & Disease & 2 & 0.583 & Disease & 4 & 0.687 & $\begin{array}{c}0.70 \\
(86 \%)\end{array}$ & Disease \\
\hline rs199617267 & I936T & Disease & 6 & 0.794 & Disease & 8 & 0.879 & $\begin{array}{c}0.86 \\
(91 \%) \\
\end{array}$ & Disease \\
\hline $\begin{array}{l}\text { rs121832180 } \\
3\end{array}$ & A907T & Disease & 1 & 0.534 & Disease & 3 & 0.635 & $\begin{array}{c}0.86 \\
(91 \%) \\
\end{array}$ & Disease \\
\hline rs199679232 & Y898C & Disease & 7 & 0.851 & Disease & 8 & 0.895 & $\begin{array}{c}0.83 \\
(90 \%) \\
\end{array}$ & Disease \\
\hline rs752349406 & N888Y & Disease & 2 & 0.599 & Disease & 5 & 0.749 & $\begin{array}{c}0.59 \\
(82 \%) \\
\end{array}$ & Disease \\
\hline $\begin{array}{l}\text { rs127012647 } \\
7\end{array}$ & D886V & Disease & 6 & 0.783 & Disease & 7 & 0.855 & $\begin{array}{c}0.81 \\
(89 \%)\end{array}$ & Disease \\
\hline $\begin{array}{l}\text { rs118186297 } \\
6\end{array}$ & Y797C & Disease & 4 & 0.686 & Disease & 5 & 0.742 & $\begin{array}{c}0.86 \\
(91 \%) \\
\end{array}$ & Disease \\
\hline rs201596987 & T793R & Disease & 3 & 0.646 & Disease & 2 & 0.589 & $\begin{array}{c}0.76 \\
(88 \%)\end{array}$ & Disease \\
\hline- & D791V & Disease & 4 & 0.695 & Disease & 5 & 0.732 & $\begin{array}{c}0.81 \\
(89 \%)\end{array}$ & Disease \\
\hline $\begin{array}{l}\text { rs145280905 } \\
1\end{array}$ & R749K & Disease & 1 & 0.562 & Disease & 3 & 0.652 & $\begin{array}{c}0.58 \\
(82 \%)\end{array}$ & Disease \\
\hline rs370575901 & $\mathrm{R} 722 \mathrm{Q}$ & Disease & 7 & 0.829 & Disease & 8 & 0.9 & $\begin{array}{c}0.69 \\
(85 \%) \\
\end{array}$ & Disease \\
\hline rs767003999 & L716S & Disease & 5 & 0.757 & Disease & 7 & 0.848 & $\begin{array}{c}0.81 \\
(89 \%) \\
\end{array}$ & Disease \\
\hline $\begin{array}{l}\text { rs127506565 } \\
4\end{array}$ & Q710R & Disease & 4 & 0.701 & Disease & 4 & 0.69 & $\begin{array}{c}0.75 \\
(87 \%)\end{array}$ & Disease \\
\hline rs763692410 & R693W & Disease & 3 & 0.63 & Disease & 8 & 0.899 & $\begin{array}{c}0.86 \\
(91 \%) \\
\end{array}$ & Disease \\
\hline rs201742754 & R689Q & Disease & 4 & 0.707 & Disease & 7 & 0.872 & $\begin{array}{c}0.70 \\
(86 \%)\end{array}$ & Disease \\
\hline
\end{tabular}




\begin{tabular}{|c|c|c|c|c|c|c|c|c|c|}
\hline rs201390288 & R689W & Disease & 5 & 0.728 & Disease & 8 & 0.911 & $\begin{array}{c}0.84 \\
(90 \%) \\
\end{array}$ & Disease \\
\hline rs56229130 & G576D & Disease & 6 & 0.821 & Disease & 7 & 0.87 & $\begin{array}{c}0.60 \\
(83 \%) \\
\end{array}$ & Disease \\
\hline rs375957332 & P410L & Disease & 6 & 0.804 & Disease & 7 & 0.859 & $\begin{array}{c}0.88 \\
(92 \%)\end{array}$ & Disease \\
\hline $\begin{array}{l}\text { rs141406192 } \\
6\end{array}$ & G396V & Disease & 6 & 0.819 & Disease & 9 & 0.932 & $\begin{array}{c}0.86 \\
(91 \%) \\
\end{array}$ & Disease \\
\hline rs775321513 & L336S & Disease & 2 & 0.612 & Disease & 2 & 0.589 & $\begin{array}{c}0.67 \\
(85 \%)\end{array}$ & Disease \\
\hline rs764033545 & H329Q & Disease & 6 & 0.813 & Disease & 8 & 0.9 & $\begin{array}{c}0.59 \\
(82 \%)\end{array}$ & Disease \\
\hline rs750663365 & Y328S & Disease & 7 & 0.829 & Disease & 8 & 0.881 & $\begin{array}{c}0.84 \\
(90 \%)\end{array}$ & Disease \\
\hline- & Y328C & Disease & 7 & 0.834 & Disease & 8 & 0.922 & $\begin{array}{c}0.80 \\
(89 \%)\end{array}$ & Disease \\
\hline- & Y328F & Disease & 2 & 0.592 & Disease & 7 & 0.829 & $\begin{array}{c}0.62 \\
(83 \%)\end{array}$ & Disease \\
\hline $\begin{array}{l}\text { rs115923559 } \\
5\end{array}$ & W323R & Disease & 8 & 0.91 & Disease & 9 & 0.939 & $\begin{array}{c}0.85 \\
(91 \%)\end{array}$ & Disease \\
\hline $\begin{array}{l}\text { rs128870697 } \\
2\end{array}$ & S298F & Disease & 6 & 0.787 & Disease & 7 & 0.866 & $\begin{array}{c}0.86 \\
(91 \%) \\
\end{array}$ & Disease \\
\hline $\begin{array}{l}\text { rs133959447 } \\
5\end{array}$ & G272R & Disease & 7 & 0.843 & Disease & 8 & 0.906 & $\begin{array}{c}0.85 \\
(91 \%)\end{array}$ & Disease \\
\hline $\begin{array}{l}\text { rs139446877 } \\
0\end{array}$ & P248L & Disease & 4 & 0.711 & Disease & 2 & 0.589 & $\begin{array}{c}0.80 \\
(89 \%)\end{array}$ & Disease \\
\hline $\begin{array}{l}\text { rs131263800 } \\
5\end{array}$ & V223E & Disease & 6 & 0.813 & Disease & 6 & 0.821 & $\begin{array}{c}0.83 \\
(90 \%) \\
\end{array}$ & Disease \\
\hline rs749382362 & $\mathrm{R} 219 \mathrm{Q}$ & Disease & 3 & 0.667 & Disease & 7 & 0.864 & $\begin{array}{c}0.64 \\
(84 \%) \\
\end{array}$ & Disease \\
\hline rs374238430 & R219W & Disease & 5 & 0.773 & Disease & 8 & 0.909 & $\begin{array}{c}0.67 \\
(85 \%) \\
\end{array}$ & Disease \\
\hline $\begin{array}{l}\text { rs103983449 } \\
2\end{array}$ & V209E & Disease & 7 & 0.839 & Disease & 7 & 0.844 & $\begin{array}{c}0.79 \\
(89 \%)\end{array}$ & Disease \\
\hline rs747349523 & D203Y & Disease & 8 & 0.893 & Disease & 9 & 0.949 & $\begin{array}{c}0.86 \\
(91 \%) \\
\end{array}$ & Disease \\
\hline rs776300430 & G202E & Disease & 6 & 0.783 & Disease & 7 & 0.843 & $\begin{array}{c}0.66 \\
(85 \%)\end{array}$ & Disease \\
\hline rs367552387 & R201Q & Disease & 5 & 0.766 & Disease & 6 & 0.792 & $\begin{array}{c}0.80 \\
(89 \%)\end{array}$ & Disease \\
\hline & R201P & Disease & 7 & 0.865 & Disease & 8 & 0.883 & $\begin{array}{c}0.86 \\
(91 \%)\end{array}$ & Disease \\
\hline
\end{tabular}




\begin{tabular}{|l|l|l|r|r|l|l|l|l|l|} 
rs773117166 & R201W & Disease & 6 & 0.787 & Disease & 7 & 0.845 & $\begin{array}{c}0.86 \\
(91 \%)\end{array}$ & Disease \\
\hline rs565257577 & G162R & Disease & 6 & 0.823 & Disease & 7 & 0.866 & $\begin{array}{c}0.84 \\
(90 \%)\end{array}$ & Disease \\
\hline
\end{tabular}

Table (3): stability analysis predicted by I-Mutant version 3.0 (Also show Most deleterious nsSNPs):

\begin{tabular}{|l|c|c|c|c|}
\hline dbSNP rs\# & $\begin{array}{c}\text { Amino } \\
\text { Acid } \\
\text { Change }\end{array}$ & $\begin{array}{c}\text { SVM2 } \\
\text { Prediction } \\
\text { Effect }\end{array}$ & RI & $\begin{array}{c}\text { DDG } \\
\text { Value } \\
\text { Prediction }\end{array}$ \\
\hline rs377679012 & R1137C & Decrease & 5 & -0.93 \\
\hline rs372723869 & Y992C & Increase & 4 & -0.46 \\
\hline rs924119518 & Y943C & Increase & 1 & -0.98 \\
\hline rs1319436059 & Y943N & Decrease & 6 & -1.34 \\
\hline rs199617267 & I936T & Decrease & 6 & -2.16 \\
\hline rs1218321803 & A907T & Decrease & -0.7 & 6 \\
\hline rs199679232 & Y898C & Decrease & -1.14 & 4 \\
\hline rs752349406 & N888Y & Increase & 0.13 & 2 \\
\hline rs1270126477 & D886V & Decrease & 0.04 & 4 \\
\hline rs1181862976 & Y797C & Increase & -0.85 & 4 \\
\hline rs201596987 & T793R & Increase & 0.02 & 4 \\
\hline- & D791V & Increase & 0.63 & 2 \\
\hline rs1452809051 & R749K & Decrease & -0.9 & 8 \\
\hline rs370575901 & R722Q & Decrease & -0.88 & 9 \\
\hline rs767003999 & L716S & Decrease & 9 & -2.22 \\
\hline rs1275065654 & Q710R & Decrease & 1 & -0.2 \\
\hline rs763692410 & R693W & Decrease & 5 & -0.48 \\
\hline rs201742754 & R689Q & Decrease & 9 & -1.24 \\
\hline rs201390288 & R689W & Decrease & 6 & -0.66 \\
\hline rs56229130 & G576D & decrease & 6 & -1.02 \\
\hline rs375957332 & P410L & decrease & 8 & -1.6 \\
\hline rs1414061926 & G396V & decrease & 8 & -0.84 \\
\hline rs775321513 & L336S & decrease & 8 & -1.89 \\
\hline rs764033545 & H329Q & decrease & 3 & -0.28 \\
\hline rs750663365 & Y328S & decrease & 7 & -0.41 \\
\hline rs1159235595 & W323R & Increase & 8 & -1.11 \\
\hline rs1288706972 & S298F & decrease & 2 & -0.13 \\
\hline
\end{tabular}




\begin{tabular}{|l|c|l|c|c|}
\hline rs1339594475 & G272R & Decrease & -0.39 & 1 \\
\hline rs1394468770 & P248L & Decrease & -0.49 & 5 \\
\hline rs1312638005 & V223E & Decrease & -1.17 & 6 \\
\hline rs749382362 & R219Q & Decrease & -1.16 & 9 \\
\hline rs374238430 & R219W & Decrease & -0.55 & 6 \\
\hline rs1039834492 & V209E & decrease & 8 & -1.18 \\
\hline rs747349523 & D203Y & increase & 0 & -0.17 \\
\hline rs776300430 & G202E & decrease & 2 & -0.51 \\
\hline rs367552387 & R201P & decrease & 6 & -0.72 \\
\hline & R201Q & decrease & 8 & -0.93 \\
\hline rs773117166 & R201W & decrease & 4 & -0.22 \\
\hline rs565257577 & G162R & decrease & 3 & -0.36 \\
\hline
\end{tabular}

Table (4): The IKBKAP gene functions and its appearance in network and genome:

\begin{tabular}{|l|c|c|c|}
\hline Function & FDR & $\begin{array}{c}\text { Genes in } \\
\text { network }\end{array}$ & $\begin{array}{c}\text { Genes in } \\
\text { genome }\end{array}$ \\
\hline transcription elongation factor complex & 0.000385 & 4 & 28 \\
\hline transcription elongation from RNA polymerase II promoter & 0.009942 & 4 & 75 \\
\hline DNA-directed RNA polymerase II, holoenzyme & 0.009942 & 4 & 81 \\
\hline nuclear DNA-directed RNA polymerase complex & 0.009942 & 4 & 95 \\
\hline RNA polymerase complex & 0.009942 & 4 & 96 \\
\hline DNA-directed RNA polymerase complex & 0.009942 & 4 & 95 \\
\hline DNA-templated transcription, elongation & 0.013634 & 4 & 108 \\
\hline positive regulation of locomotion & 0.125453 & 4 & 213 \\
\hline positive regulation of cell motility & 0.125453 & 4 & 204 \\
\hline positive regulation of cell migration & 0.125453 & 4 & 199 \\
\hline
\end{tabular}

*FDR: false discovery rate is greater than or equal to the probability that this is a false positive.

Table (5) The gene co-expressed, share domain and Interaction with IKBKAP gene network:(Also show The 41 novel mutations):

\begin{tabular}{|c|c|c|c|}
\hline Gene 1 & Gene 2 & Weight & Network group \\
\hline ELP2 & ELP1 & 0.009 & Co-expression \\
\hline GAPVD1 & ELP1 & 0.010505 & Co-expression \\
\hline URM1 & PPP6C & 0.012673 & Co-expression \\
\hline GAPVD1 & ELP1 & 0.019938 & Co-expression \\
\hline CD2AP & ELP1 & 0.010033 & Co-expression \\
\hline URM1 & PPP6C & 0.014834 & Co-expression \\
\hline
\end{tabular}




\begin{tabular}{|c|c|c|c|}
\hline GAPVD1 & ELP1 & 0.020476 & Co-expression \\
\hline GAPVD1 & PPP6C & 0.017376 & Co-expression \\
\hline FOCAD & ELP1 & 0.010588 & Co-expression \\
\hline IKBKB & ELP1 & 0.009126 & Co-expression \\
\hline ELP5 & ELP6 & 0.017894 & Co-expression \\
\hline IRF4 & NFKBIA & 0.017726 & Co-expression \\
\hline ELP2 & ELP1 & 0.006755 & Co-expression \\
\hline DMAP1 & ELP1 & 0.013834 & Co-expression \\
\hline ELP4 & PPP6C & 0.009147 & Co-expression \\
\hline IRF4 & NFKBIA & 0.013234 & Co-expression \\
\hline NFKBIA & ELP1 & 0.315041 & Pathway \\
\hline IKBKB & NFKBIA & 0.012293 & Pathway \\
\hline MAP3K14 & IKBKB & 0.013495 & Pathway \\
\hline SHC1 & ELP1 & 0.126671 & Physical Interactions \\
\hline ELP3 & ELP2 & 0.181392 & Physical Interactions \\
\hline TSC22D1 & ELP1 & 0.331631 & Physical Interactions \\
\hline NUP50 & ELP1 & 0.277373 & Physical Interactions \\
\hline NUP50 & TSC22D1 & 0.157924 & Physical Interactions \\
\hline CD2AP & ELP1 & 0.137789 & Physical Interactions \\
\hline CD2AP & ELP2 & 0.049176 & Physical Interactions \\
\hline CD2AP & TSC22D1 & 0.078451 & Physical Interactions \\
\hline ELP2 & ELP1 & 0.333333 & Physical Interactions \\
\hline ELP3 & ELP1 & 0.333333 & Physical Interactions \\
\hline ELP3 & ELP2 & 0.333333 & Physical Interactions \\
\hline ELP4 & ELP1 & 0.333333 & Physical Interactions \\
\hline ELP4 & ELP2 & 0.333333 & Physical Interactions \\
\hline ELP4 & ELP3 & 0.333333 & Physical Interactions \\
\hline NFKBIA & ELP1 & 0.215941 & Physical Interactions \\
\hline IKBKB & NFKBIA & 0.037527 & Physical Interactions \\
\hline URM1 & ELP1 & 0.141754 & Physical Interactions \\
\hline MAP3К14 & IKBKB & 0.048823 & Physical Interactions \\
\hline IRF4 & ELP1 & 0.407145 & Physical Interactions \\
\hline ELP2 & ELP1 & 0.133207 & Physical Interactions \\
\hline ELP3 & ELP1 & 0.231538 & Physical Interactions \\
\hline ELP3 & ELP2 & 0.344877 & Physical Interactions \\
\hline ELP4 & ELP2 & 0.48935 & Physical Interactions \\
\hline DPH3 & ELP1 & 0.517968 & Physical Interactions \\
\hline FOCAD & ELP1 & 0.517968 & Physical Interactions \\
\hline ELP2 & ELP1 & 0.0749 & Physical Interactions \\
\hline ELP3 & ELP1 & 0.083596 & Physical Interactions \\
\hline ELP3 & ELP2 & 0.1826 & Physical Interactions \\
\hline
\end{tabular}




\begin{tabular}{|c|c|c|c|}
\hline NFKBIA & ELP1 & 0.014861 & Physical Interactions \\
\hline ELP4 & ELP1 & 0.104887 & Physical Interactions \\
\hline ELP4 & ELP2 & 0.229106 & Physical Interactions \\
\hline ELP4 & ELP3 & 0.255706 & Physical Interactions \\
\hline IKBKB & ELP1 & 0.011662 & Physical Interactions \\
\hline IKBKB & NFKBIA & 0.005054 & Physical Interactions \\
\hline ELP6 & ELP1 & 0.286862 & Physical Interactions \\
\hline MAP3К14 & ELP1 & 0.038893 & Physical Interactions \\
\hline МАР3К14 & NFKBIA & 0.016857 & Physical Interactions \\
\hline MAP3К14 & IKBKB & 0.013228 & Physical Interactions \\
\hline ELP5 & ELP1 & 0.170383 & Physical Interactions \\
\hline IKBKB & ELP1 & 0.255299 & Physical Interactions \\
\hline IKBKB & NFKBIA & 0.058324 & Physical Interactions \\
\hline MAP3К14 & IKBKB & 0.643594 & Physical Interactions \\
\hline ELP6 & ELP4 & 0.312748 & Physical Interactions \\
\hline ELP5 & ELP6 & 0.312748 & Physical Interactions \\
\hline ELP3 & ELP1 & 0.252782 & Physical Interactions \\
\hline NFKBIA & ELP1 & 0.016702 & Physical Interactions \\
\hline NFKBIA & ELP3 & 0.139754 & Physical Interactions \\
\hline IRF4 & ELP1 & 0.0903 & Physical Interactions \\
\hline IKBKB & NFKBIA & 0.011146 & Physical Interactions \\
\hline МАР3К14 & IKBKB & 0.006473 & Physical Interactions \\
\hline ELP5 & ELP4 & 0.751237 & Physical Interactions \\
\hline ELP5 & ELP6 & 0.583434 & Physical Interactions \\
\hline IKBKB & ELP1 & 0.022347 & Physical Interactions \\
\hline МАР3К14 & ELP1 & 0.025064 & Physical Interactions \\
\hline MAР3К14 & IKBKB & 0.01664 & Physical Interactions \\
\hline ELP2 & ELP1 & 0.5 & Predicted \\
\hline ELP3 & ELP1 & 0.5 & Predicted \\
\hline ELP3 & ELP2 & 0.5 & Predicted \\
\hline ELP2 & ELP1 & 0.108428 & Predicted \\
\hline ELP3 & ELP1 & 0.095014 & Predicted \\
\hline ELP3 & ELP2 & 0.106134 & Predicted \\
\hline PPP6C & ELP1 & 0.040139 & Predicted \\
\hline DPH3 & ELP1 & 0.092118 & Predicted \\
\hline $\mathrm{DPH} 3$ & ELP2 & 0.1029 & Predicted \\
\hline $\mathrm{DPH} 3$ & ELP3 & 0.090169 & Predicted \\
\hline KTI12 & ELP1 & 0.161683 & Predicted \\
\hline KTI12 & ELP2 & 0.180607 & Predicted \\
\hline KTI12 & ELP3 & 0.158263 & Predicted \\
\hline DMAP1 & ELP1 & 0.092452 & Predicted \\
\hline
\end{tabular}




\begin{tabular}{|c|c|c|c|} 
PPP6C & ELP1 & 1 & Predicted \\
\hline DPH3 & ELP2 & 0.707107 & Predicted \\
\hline DPH3 & ELP3 & 0.707107 & Predicted \\
\hline ELP2 & ELP1 & 0.53965 & Predicted \\
\hline ELP4 & ELP1 & 0.53965 & Predicted \\
\hline IKBKB & ELP1 & 0.081517 & Predicted \\
\hline MAP3K14 & ELP1 & 0.126572 & Predicted \\
\hline MAP3K14 & NFKBIA & 0.068474 & Predicted \\
\hline ELP2 & ELP1 & 0.393402 & Predicted \\
\hline ELP3 & ELP1 & 0.451851 & Predicted \\
\hline ELP3 & ELP2 & 0.290279 & Predicted \\
\hline KTI12 & ELP2 & 0.66162 & Predicted \\
\hline MAP3K14 & IKBKB & 0.00439 & Shared protein domains \\
\hline
\end{tabular}

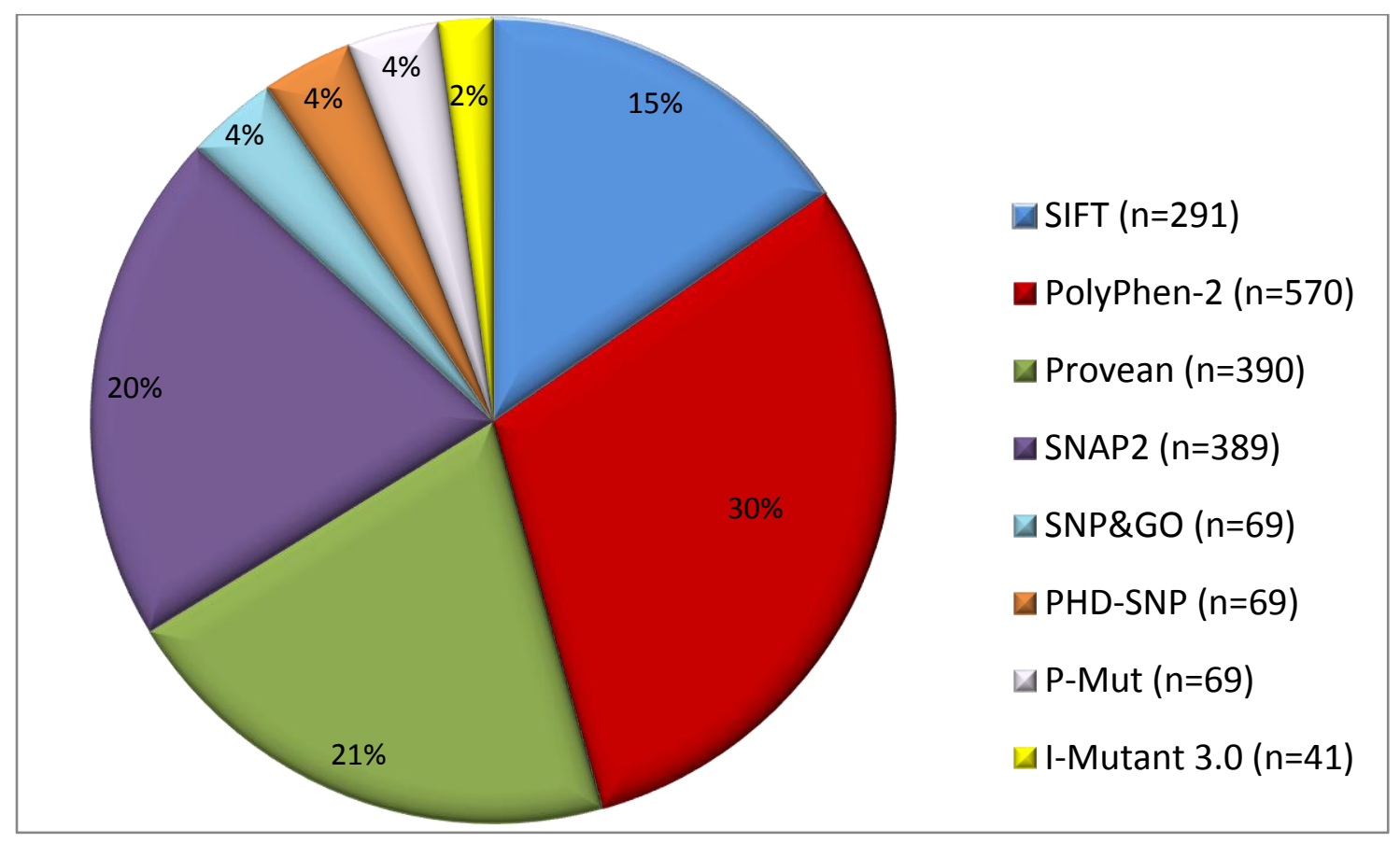

Figure 1: Pie chart showing percentage of damaging nsSNPs identified. The distribution of damaging nsSNPs by percentage (\%) and number of SNPs ( $n$ ) identified by eight in silico tools; SIFT, PolyPhen-2, Provean, SNAP2, SNP\&GO, PHD-SNP, P-MUT and I-Mutant 3.0. 
bioRxiv preprint doi: https://doi.org/10 1101/436071; this version posted October 5, 2018. The copyright holder for this preprint (which was not certified by peer review) is the author/funder, who has granted bioRxiv a license to display the preprint in perpetuity. It is made available under

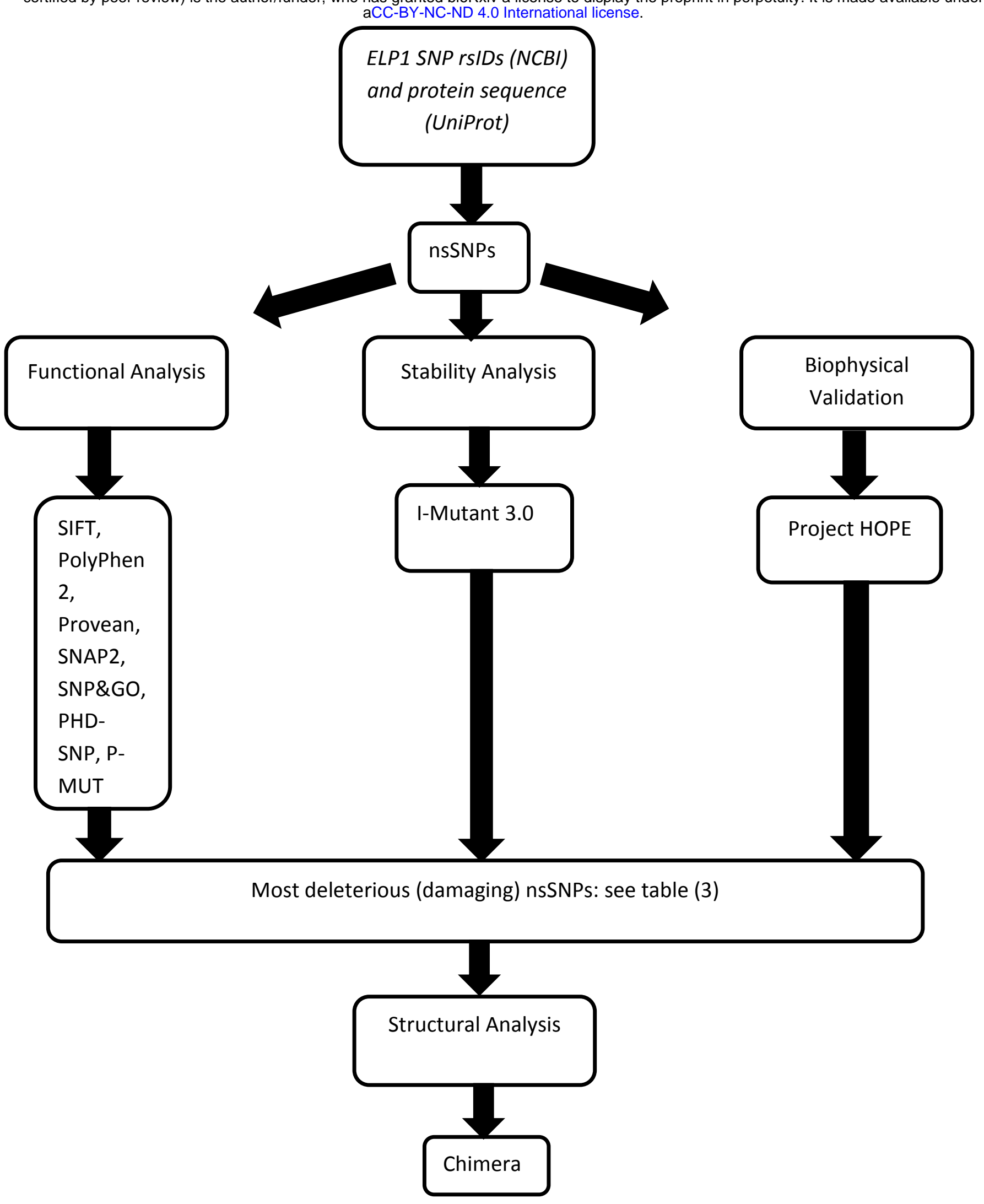

Figure 2: Diagrammatic representation of IKBKAP gene in silico work flow. 
bioRxiv preprint doi: https://doi.org/10.1101/436071; this version posted October 5, 2018. The copyright holder for this preprint (which was not certified by peer review) is the author/funder, who has granted bioRxiv a license to display the preprint in perpetuity. It is made available under aCC-BY-NC-ND 4.0 International license.

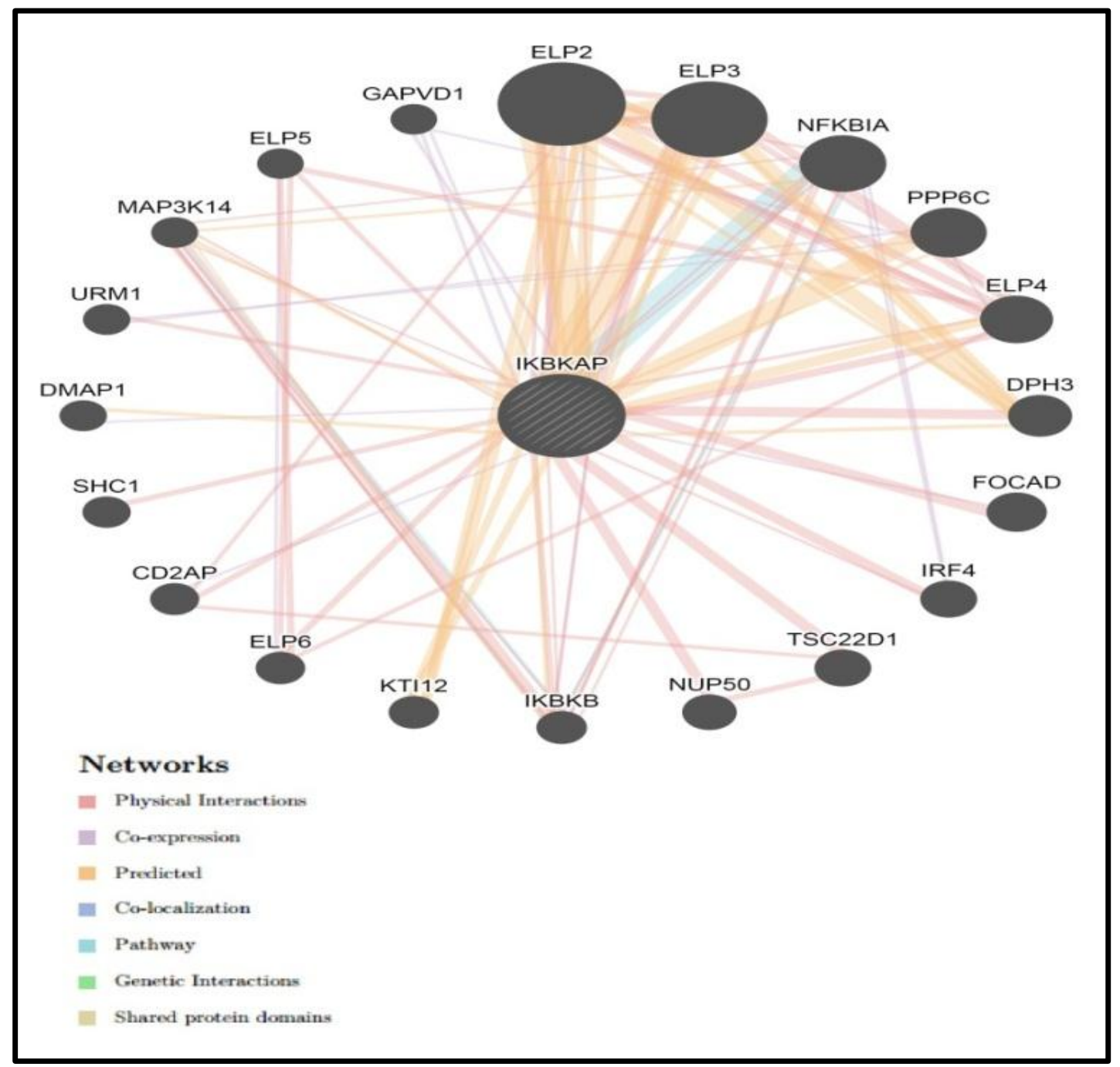

Figure 3: interaction between IKBKAP and its related genes.

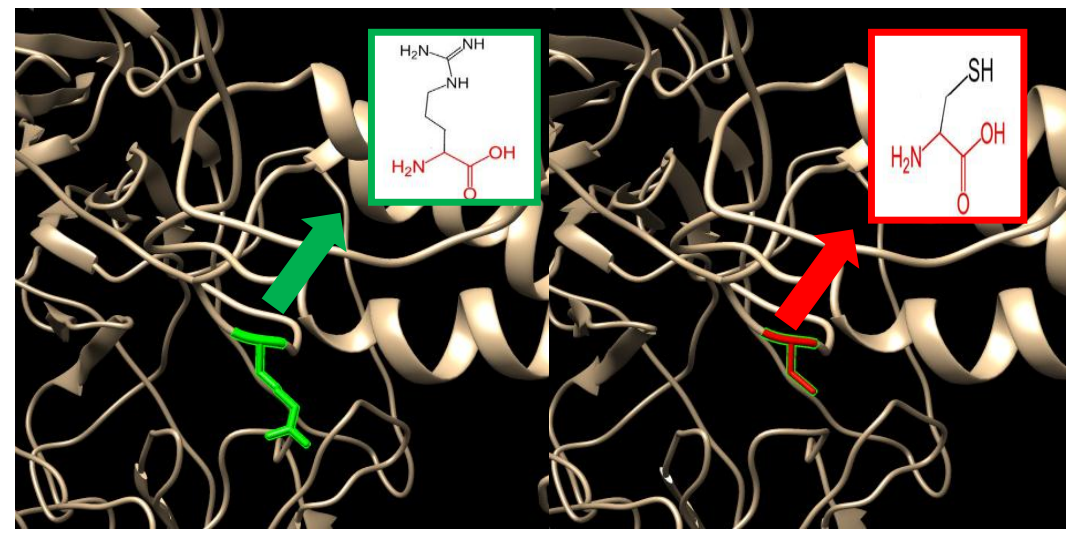

Figure 4 :( rs377679012) :( R1137C): The amino acid Arginine change to Cysteine at position 1137. 

aCC-BY-NC-ND 4.0 International license.

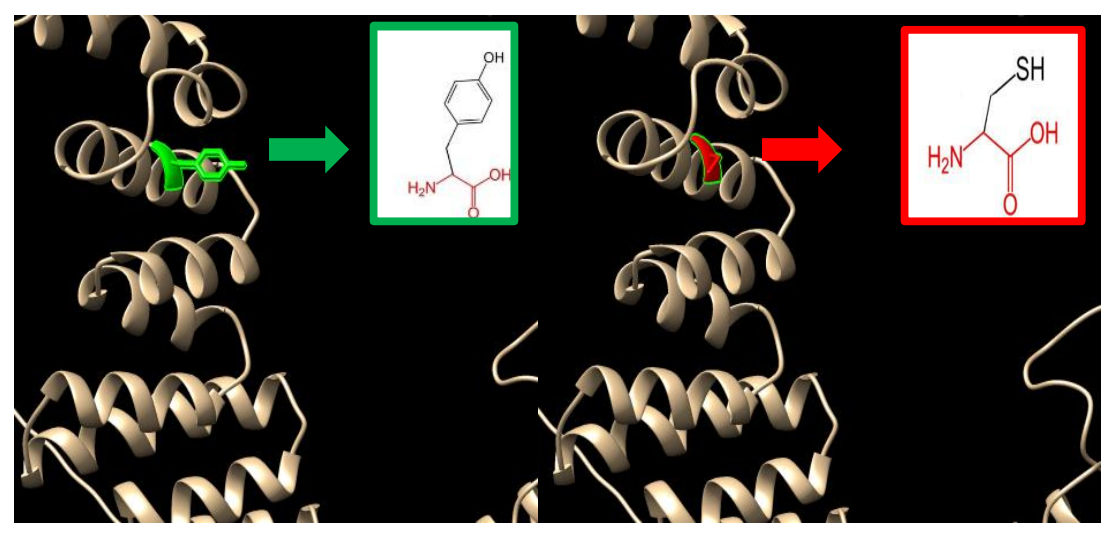

Figure 5:( rs372723869):( Y992C): The amino acid Tyrosine change to Cysteine at position 992.

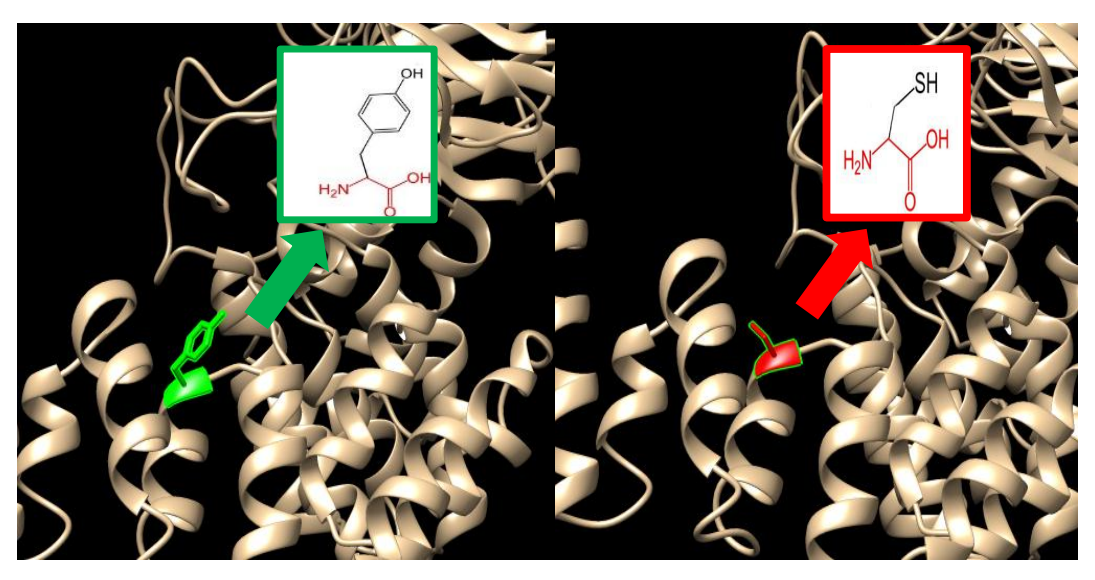

Figure 6:( rs924119518):( Y943C): The amino acid Tyrosine change to Cysteine at position 943.

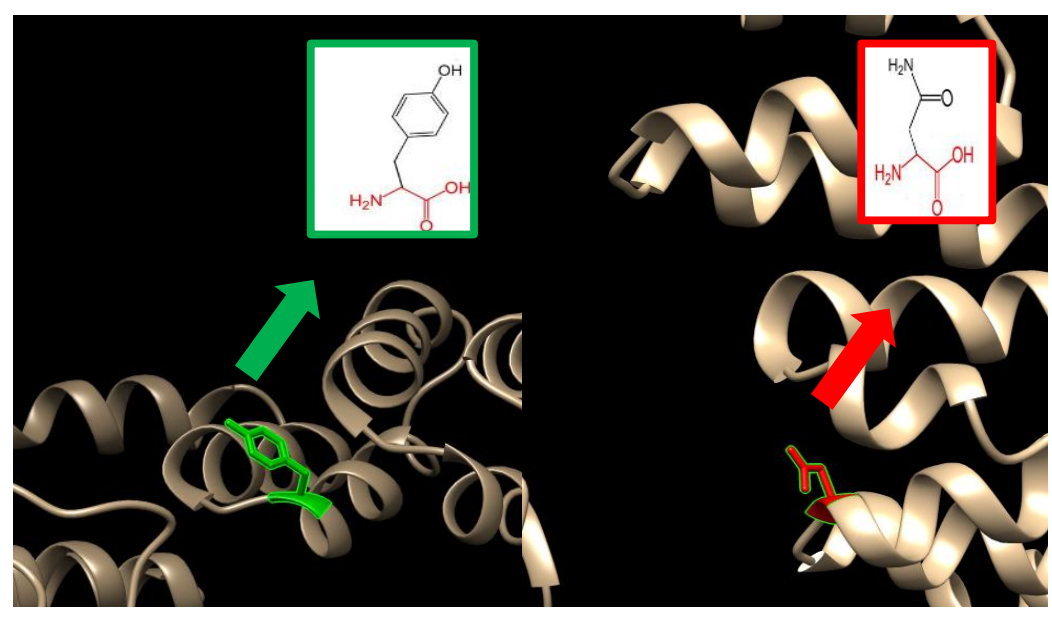

Figure $7:($ rs1319436059) :( Y943N): The amino acid Tyrosine change to Asparagine at position 943. 
bioRxiv preprint doi: https://doi.org/10.1101/436071; this version posted October 5, 2018. The copyright holder for this preprint (which was not certified by peer review) is the author/funder, who has granted bioRxiv a license to display the preprint in perpetuity. It is made available under aCC-BY-NC-ND 4.0 International license.

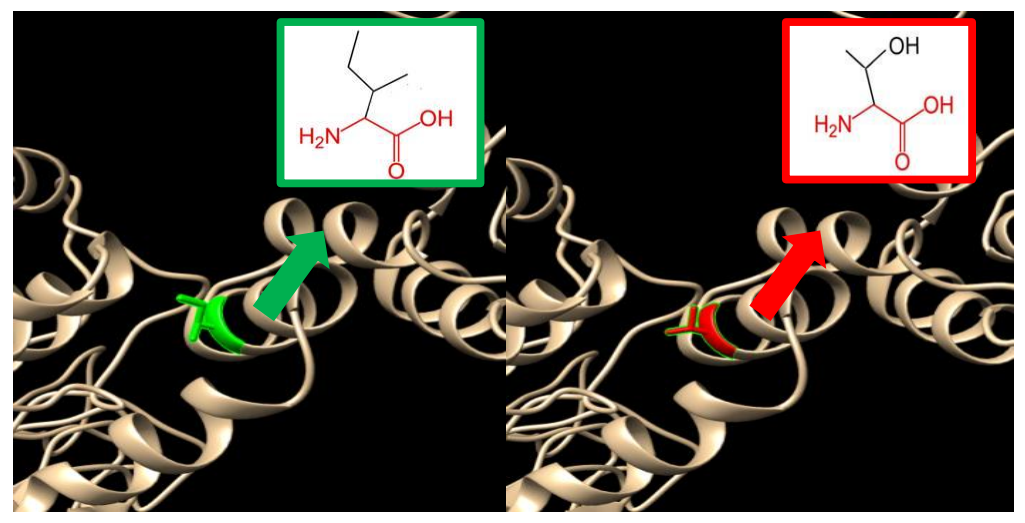

Figure 8::( rs199617267) :( 1936T): The amino acid Isoleucine change to Threonine at position 936.

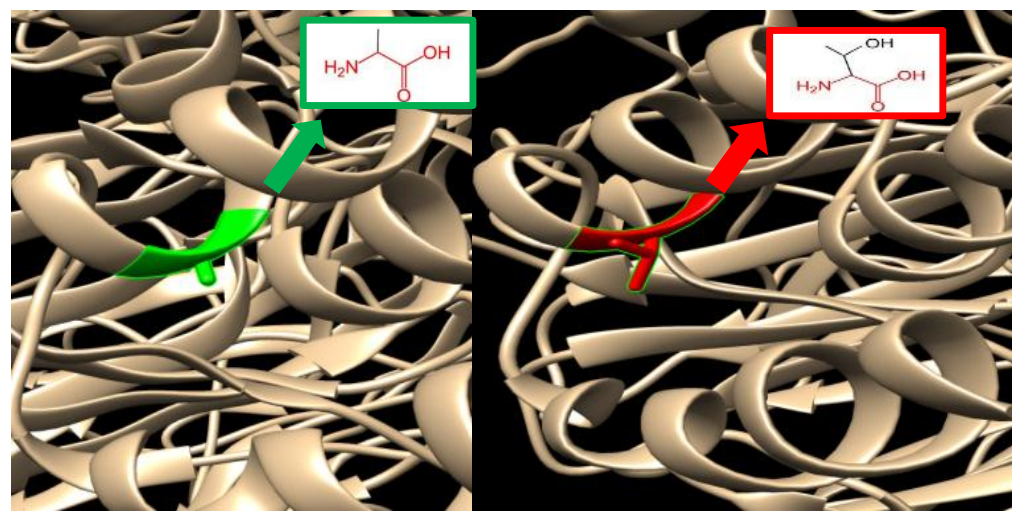

Figure 9 :(rs1218321803): (A907T): The amino acid alanine to threonine at postion907.

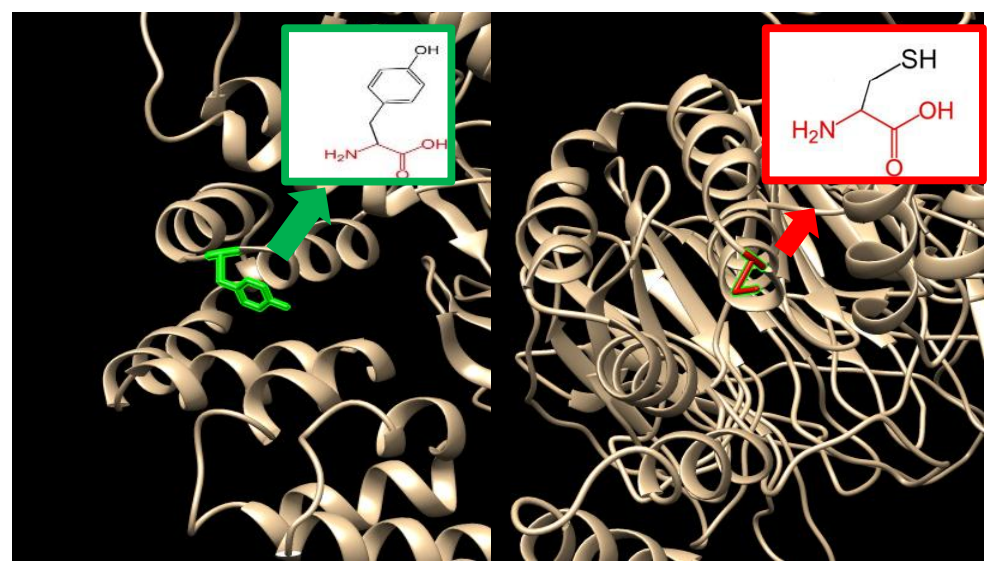

Figure 10 :(rs199679232): (Y898C): The amino acid Tyrosine change to Cysteine at position 898. 
bioRxiv preprint doi: https://doi.org/10.1101/436071; this version posted October 5,2018 . The copyright holder for this preprint (which was not certified by peer review) is the author/funder, who has granted bioRxiv a license to display the preprint in perpetuity. It is made available under

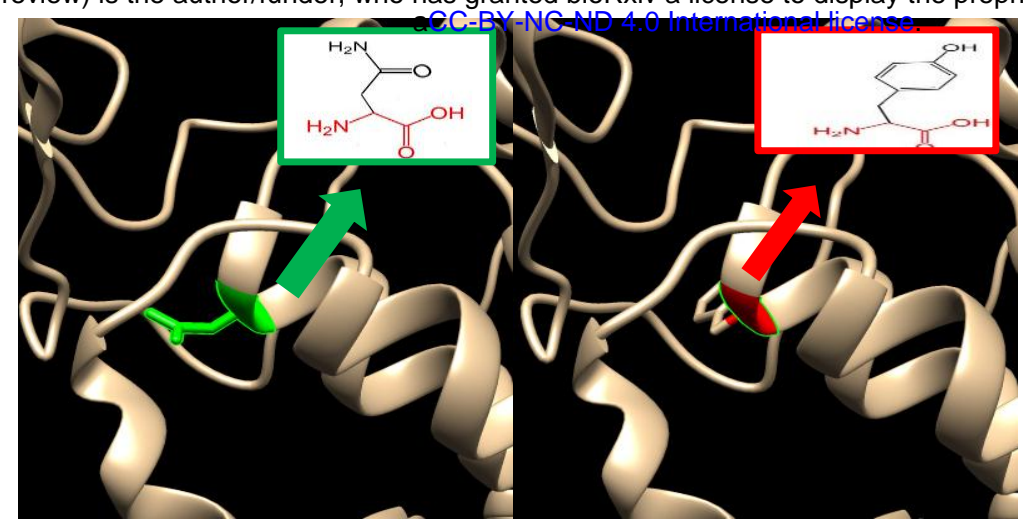

Figure 11 :(rs752349406):(N888Y)The amino acid Asparagine change toTyrosine at position 888.

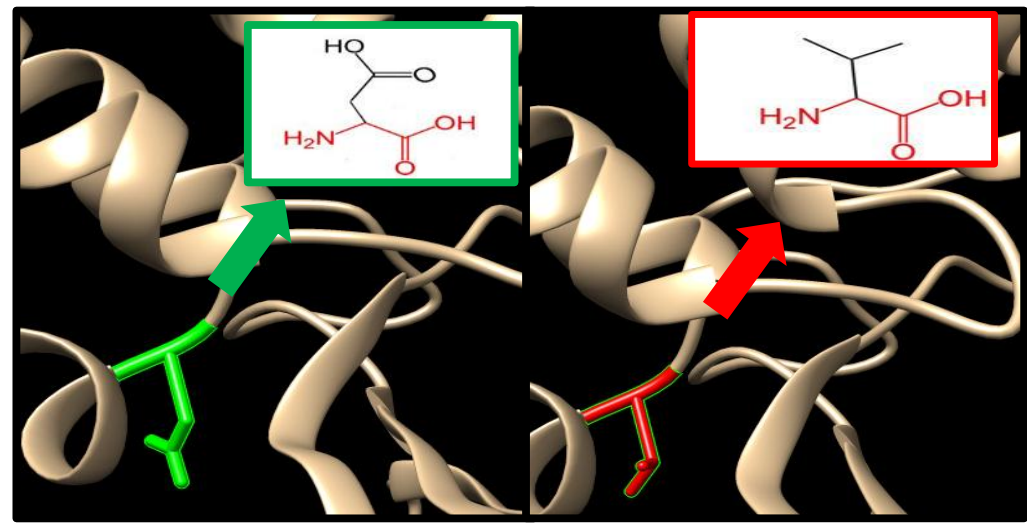

Figure 12 :(rs1270126477):(D886V) The amino acid Aspartic acid change to Valine at position 886.

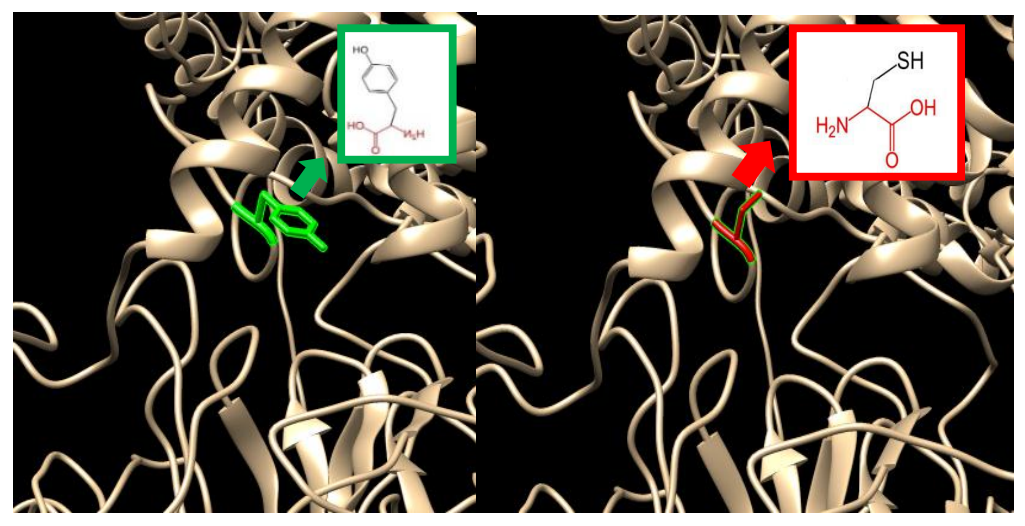

Figure 13 :( rs1181862976):( Y797C): The amino acid Tyrosine change to Cysteine at position797. 

aCC-BY-NC-ND 4.0 International license.

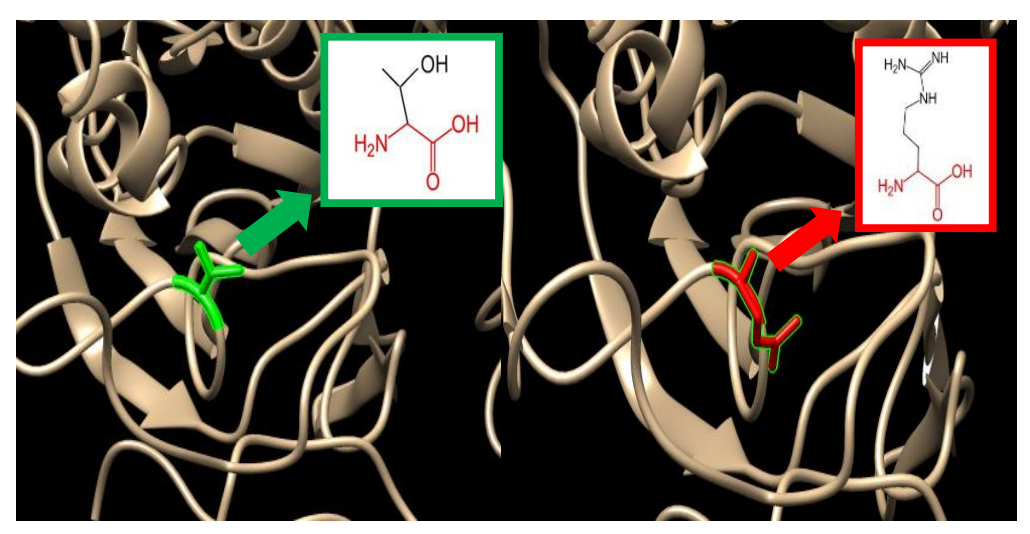

Figure 14 :( rs201596987) :( T793R): The amino acid Threonine change to Arginine at position 793.

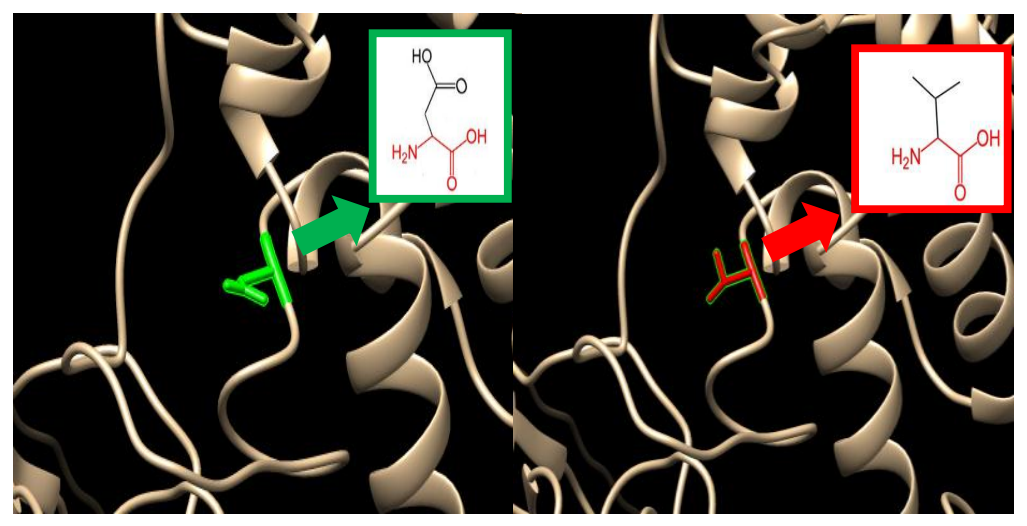

Figure 15 :( D791V): The amino acid Aspartic acid change to Valine at position 791.

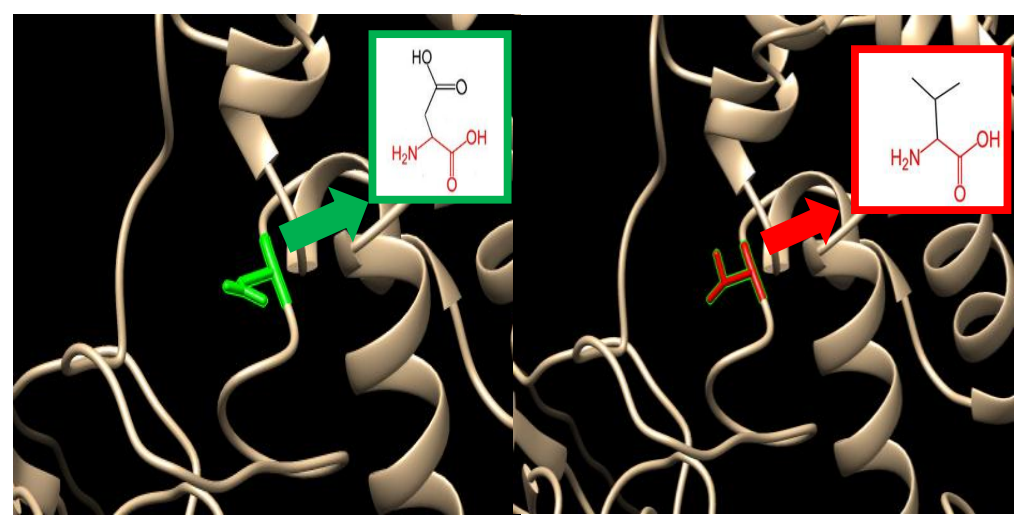

Figure 16 :( (rs1452809051):(R749K): The amino acid Arginine change to Lysine at position 749. 
bioRxiv preprint doi: https://doi.org/10.1101/436071; this version posted October 5, 2018. The copyright holder for this preprint (which was not certified by peer review) is the author/funder, who has granted bioRxiv a license to display the preprint in perpetuity. It is made available under aCC-BY-NC-ND 4.0 International license.

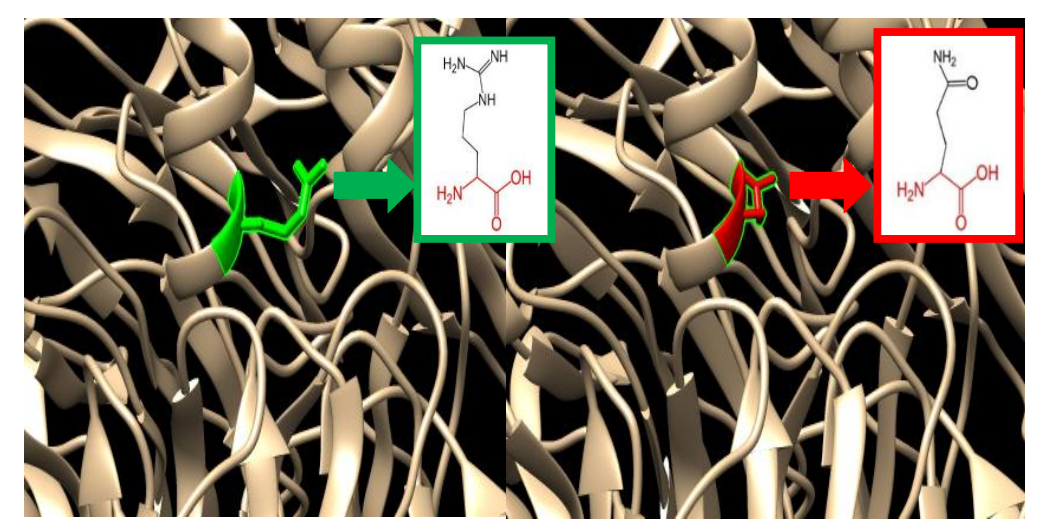

Figure 17 :( rs1452809051) (R722Q): The amino acid Arginine change to Glutamine at position 722.

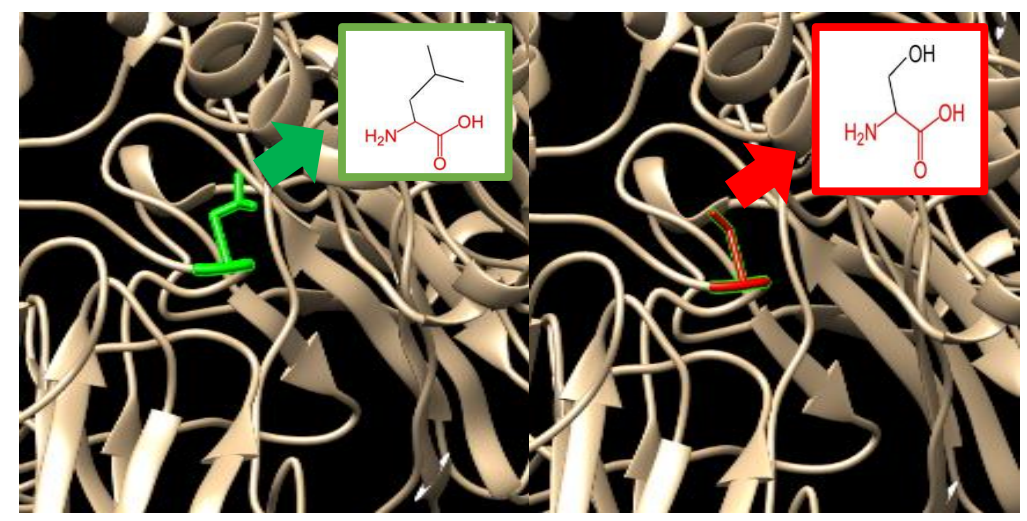

Figure 18: (rs767003999): (L716S): The amino acid Leucine change to serine at position 716.

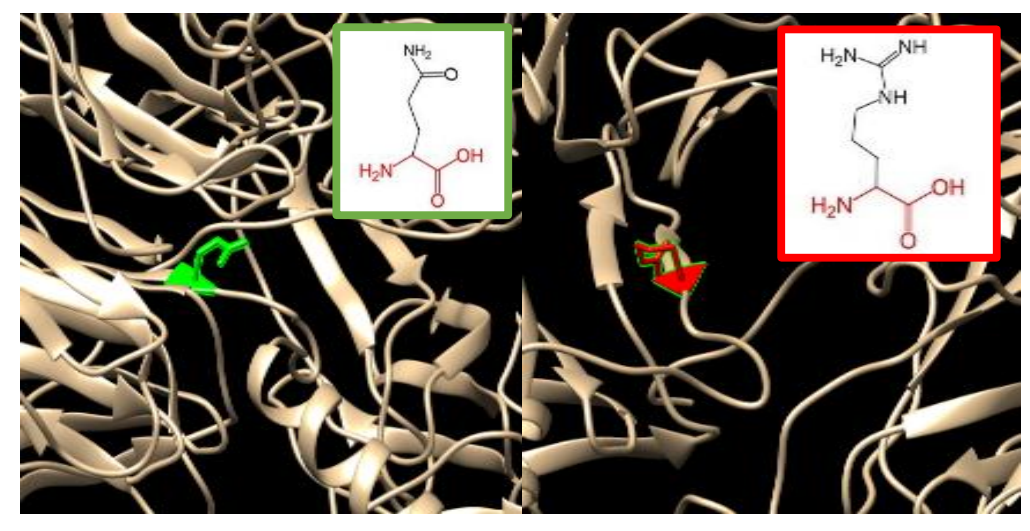

Figure 19: (rs1275065654): (Q710R): The amino acid Glutamine change to Arginine at position 710. 
bioRxiv preprint doi: https://doi.org/101101/436071; this version posted October 5, 2018. The copyright holder for this preprint (which was not certified by peer review) is the author/funder, who has granted bioRxiv a license to display the preprint in perpetuity. It is made available under

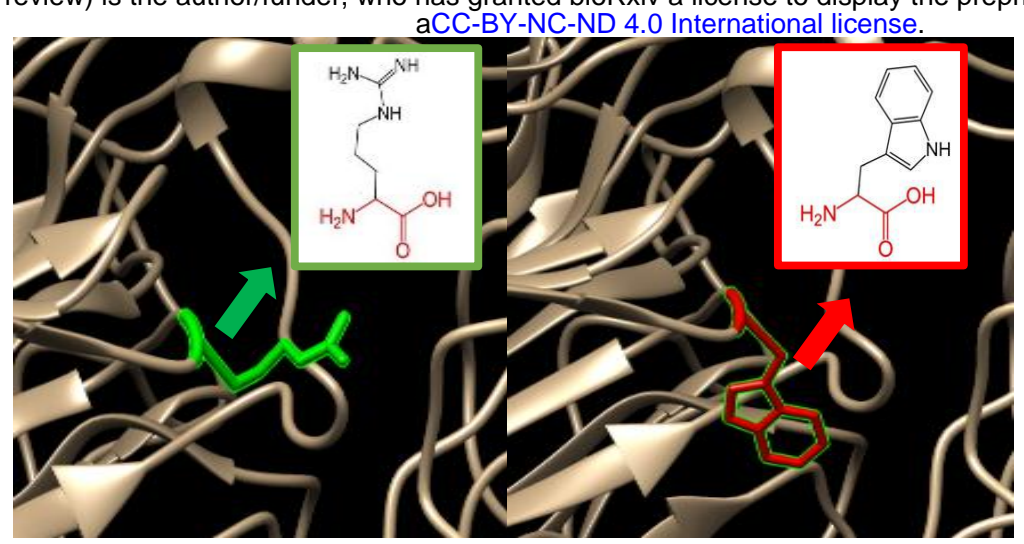

Figure 20: (rs763692410) :( R693W): The amino acid arginine change to Tryptophan at position 693.

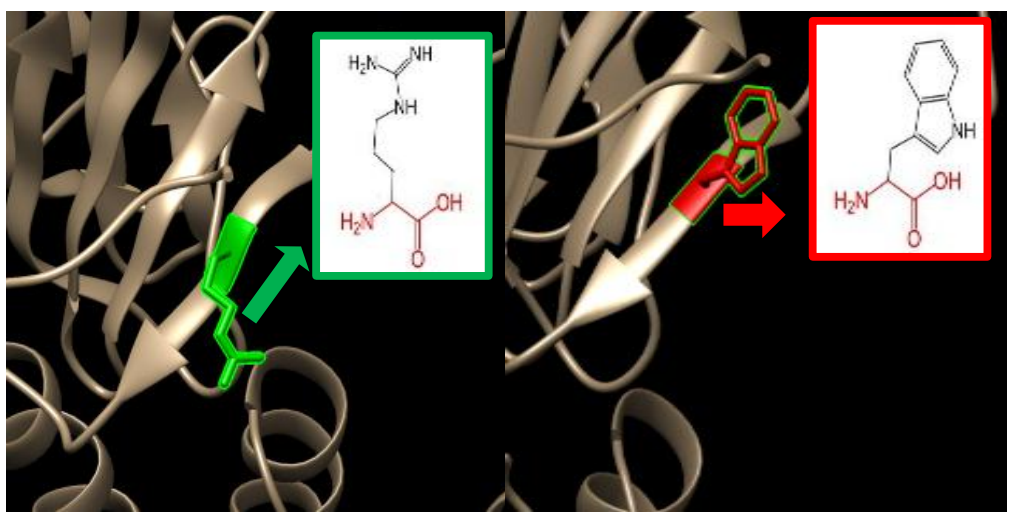

Figure 21: (rs201742754): (R689Q): The amino acid Arginine change to Glutamine at position 689.

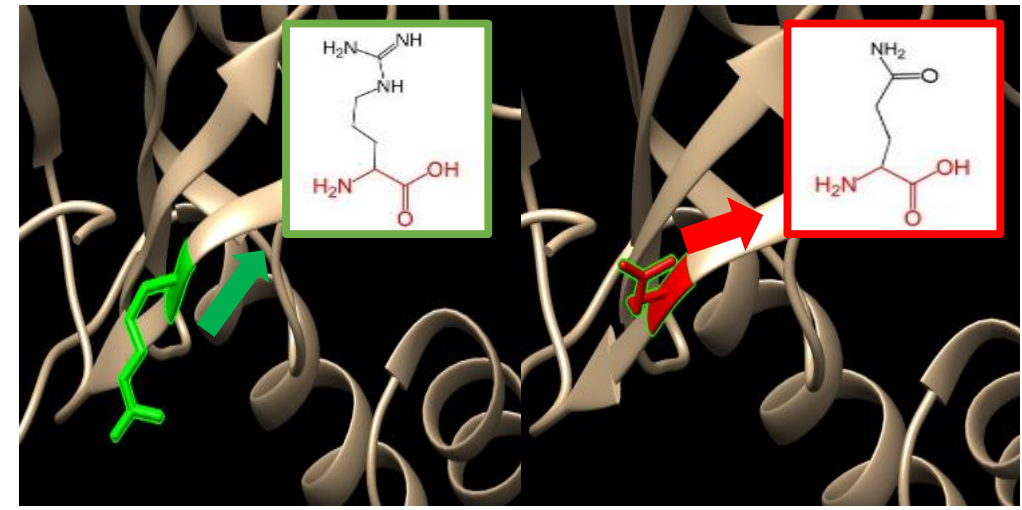

Figure 22: (rs201390288): (R689W): The amino acids Arginine change to Tryptophan at position 689. 
bioRxiv preprint doi: https://doi.org/10.1101/436071; this version posted October 5, 2018. The copyright holder for this preprint (which was not certified by peer review) is the author/funder, who has granted bioRxiv a license to display the preprint in perpetuity. It is made available under aCC-BY-NC-ND 4.0 International license.

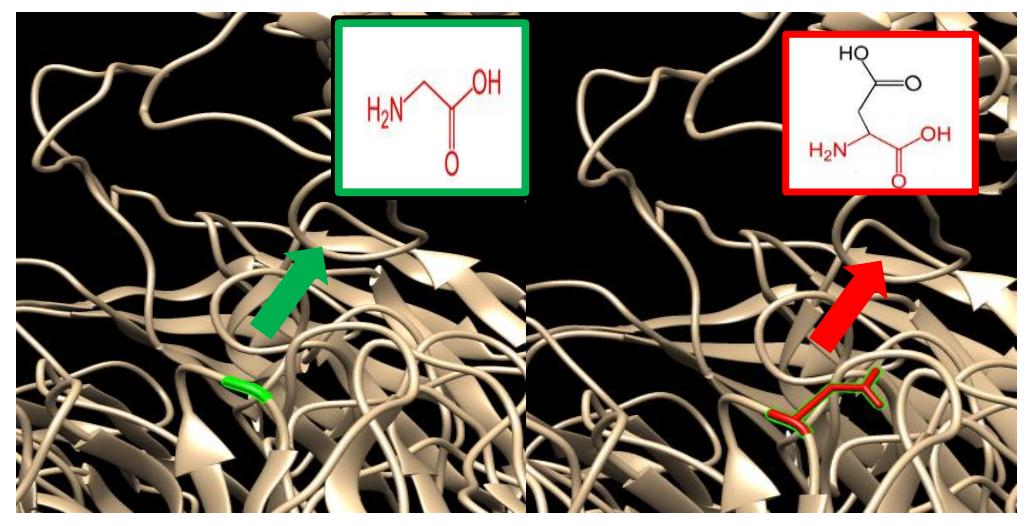

Figure 23: (rs56229130): (G576D): The amino acid Glycine changed to Aspartic acid at position 576.

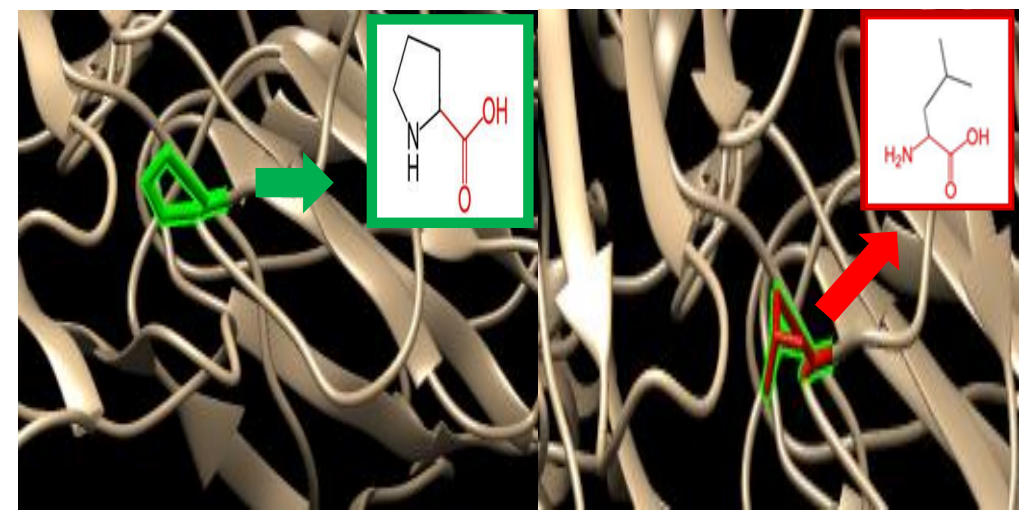

Figure 24:(rs375957332):(P410L) : The amino acid Proline changed to Leucine at position 410 .

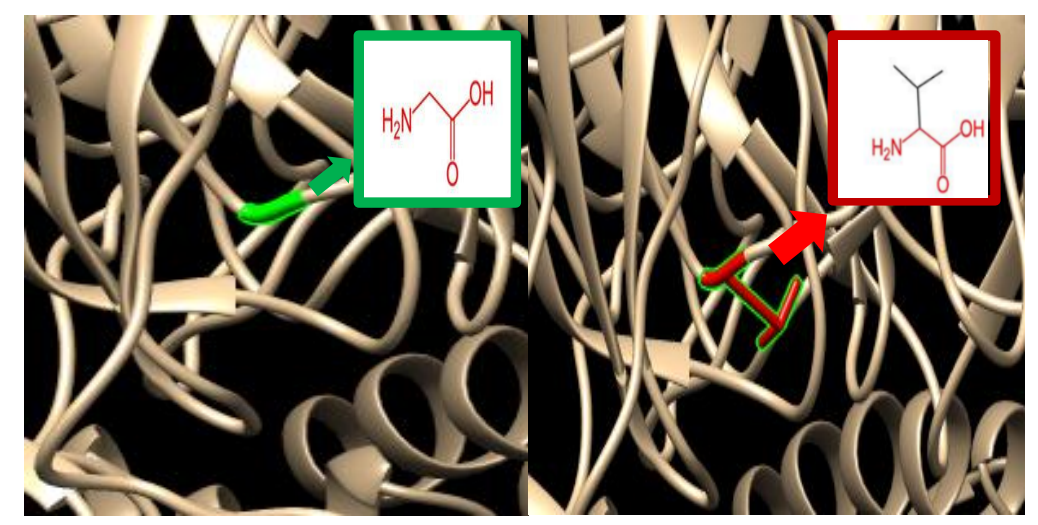

Figure 25: (rs1414061926): (G396V): The amino acid Glycine changes to Valine at position 396. 
bioRxiv preprint doi: https://doi.org/10.1101/436071; this version posted October 5, 2018. The copyright holder for this preprint (which was not certified by peer review) is the author/funder, who has granted bioRxiv a license to display the preprint in perpetuity. It is made available under aCC-BY-NC-ND 4.0 International license.

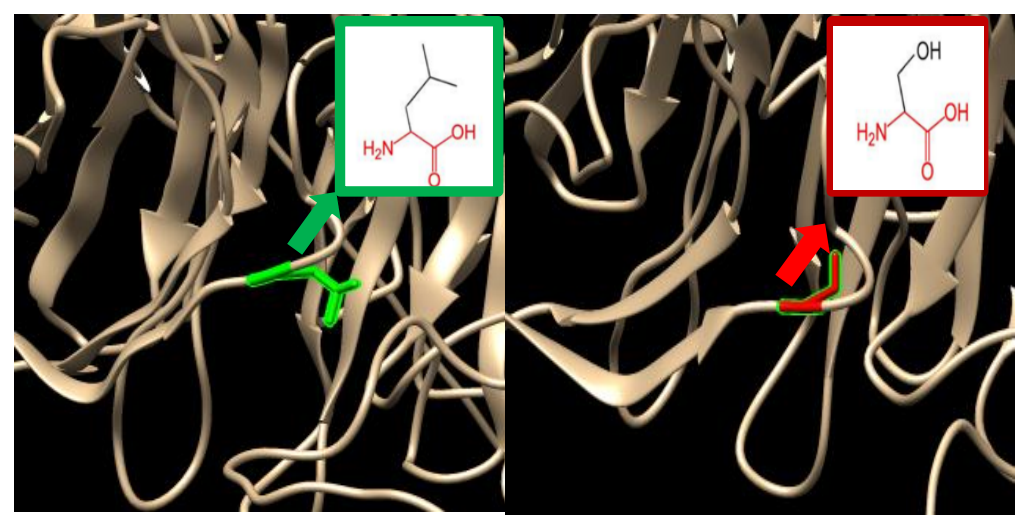

Figure 26: (rs775321513): (L336S): The amino acid Leucine changes to serine at position 336.

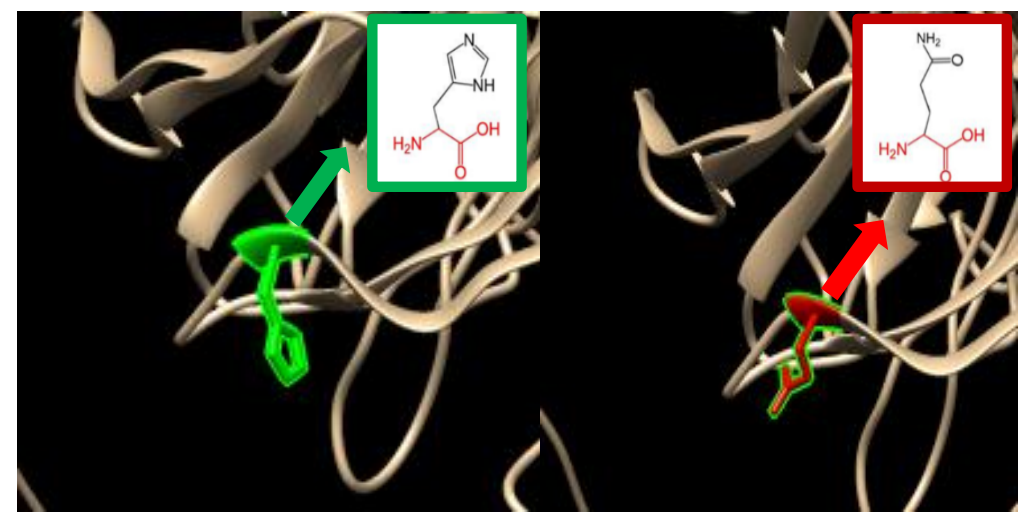

Figure 27: (rs764033545): (H329Q): The amino acids Histidine changes to glutamine at position 329.

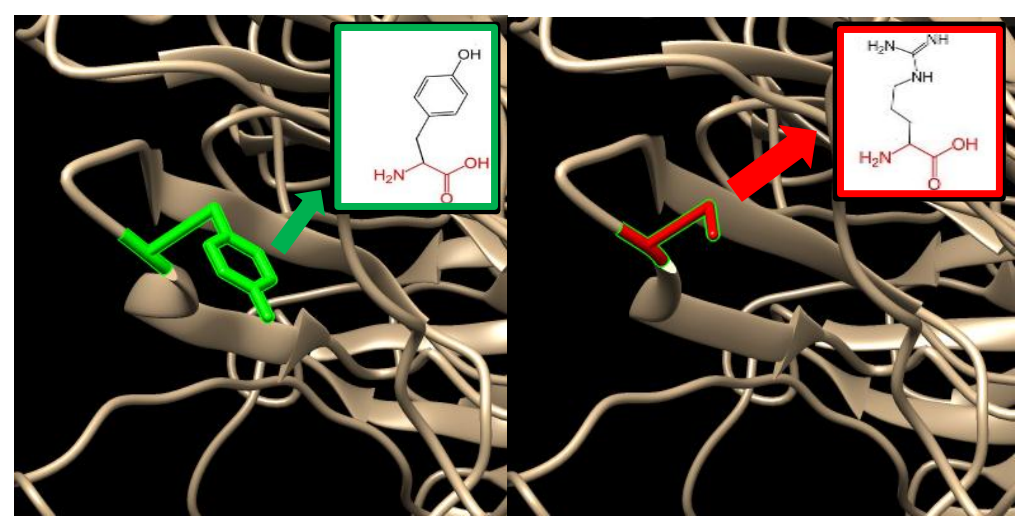

Figure 28: (rs750663365): (Y328S): The amino acid Tyrosine change to Serine at position 328. 
bioRxiv preprint doi: https://doi.org/10.1101/436071; this version posted October 5, 2018. The copyright holder for this preprint (which was not certified by peer review) is the author/funder, who has granted bioRxiv a license to display the preprint in perpetuity. It is made available under aCC-BY-NC-ND 4.0 International license.

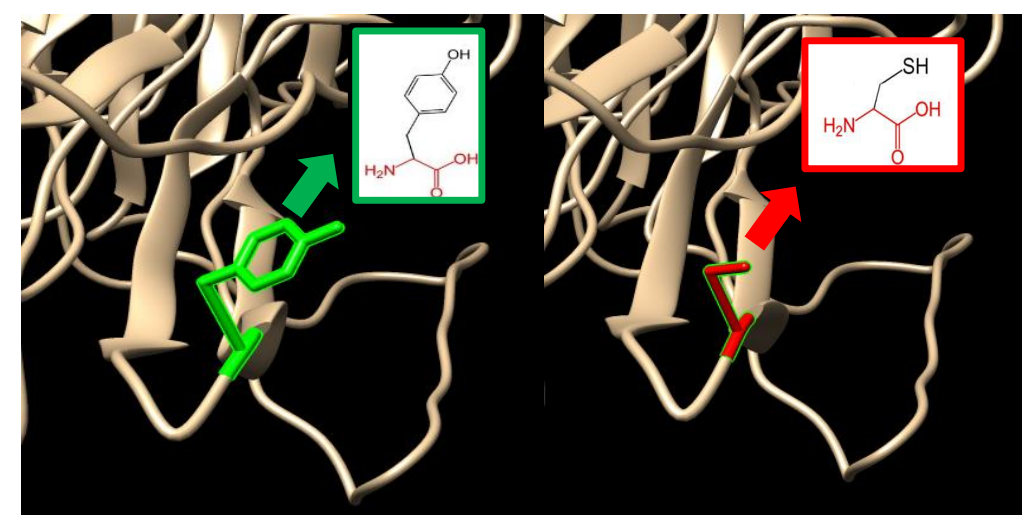

Figure 29: (Y328C): The amino acid Tyrosine change to Cysteine at position 328.

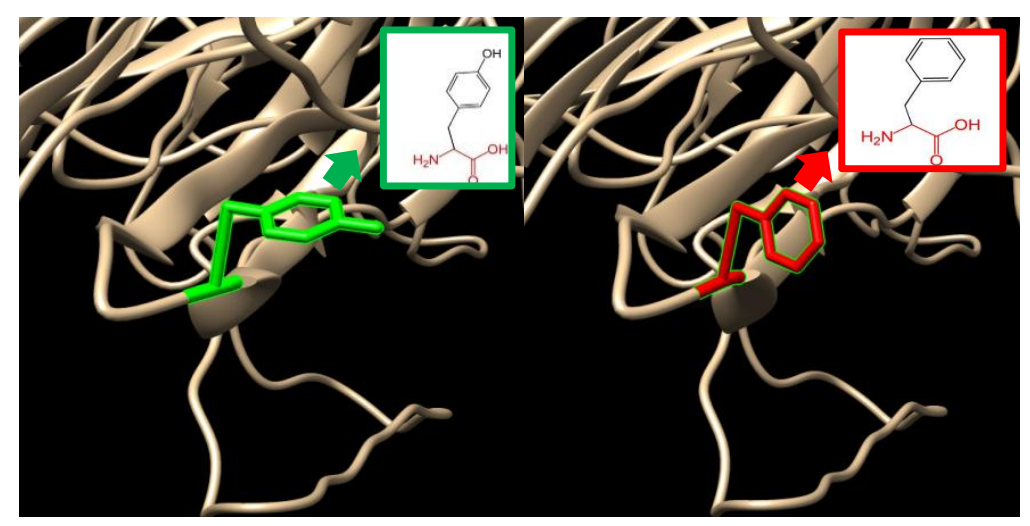

Figure 30: (Y328F): The amino acid Tyrosine changes to Phenylalanine at position 328.

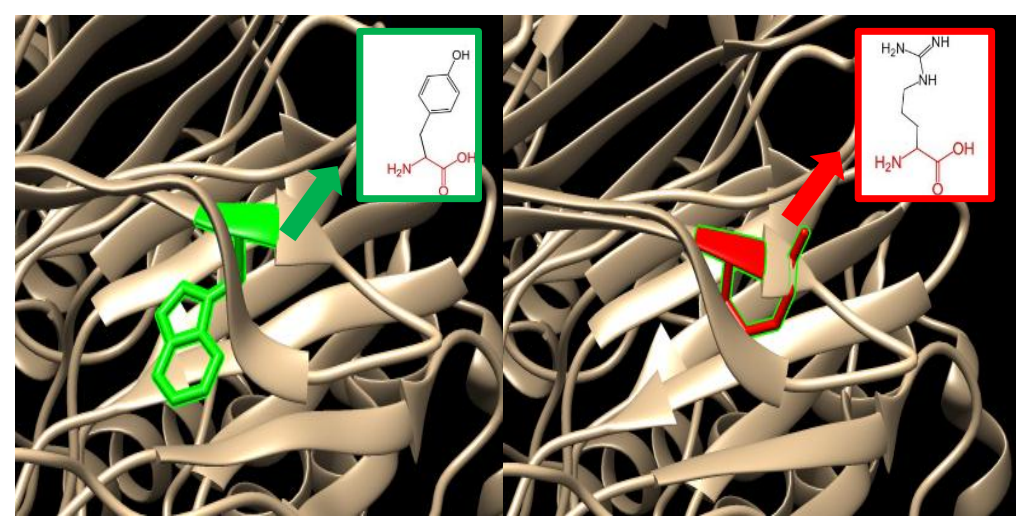

Figure 31: (rs1159235595): (W323R): The amino acid Tryptophan changes to Arginine at position 323. 


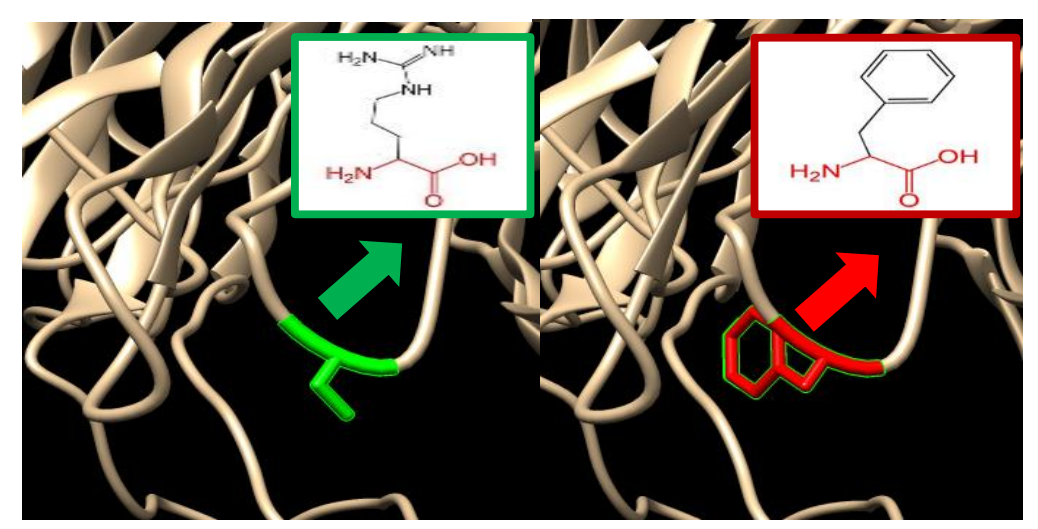

Figure 32: (rs1288706972): (S298F): The amino acid serine change to Phenylalanine at position 298.

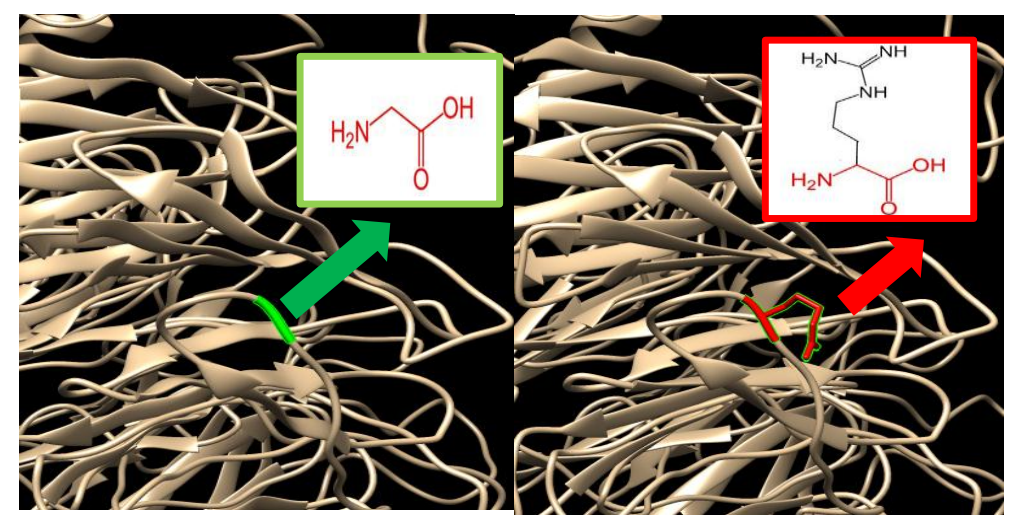

Figure 33: (rs1339594475): (G272R): The amino acid Glycine change to Arginine at position 272.

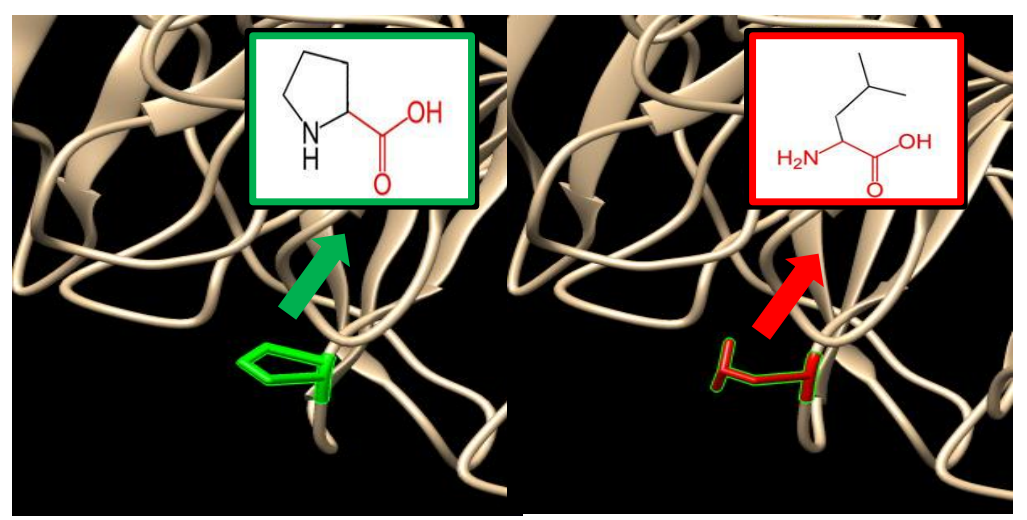

Figure 34: (rs1394468770): (P248L): The amino acid Proline changes to Leucine at position 248. 
bioRxiv preprint doi: https://doi.org/10.1101/436071; this version posted October 5, 2018. The copyright holder for this preprint (which was not certified by peer review) is the author/funder, who has granted bioRxiv a license to display the preprint in perpetuity. It is made available under aCC-BY-NC-ND 4.0 International license.

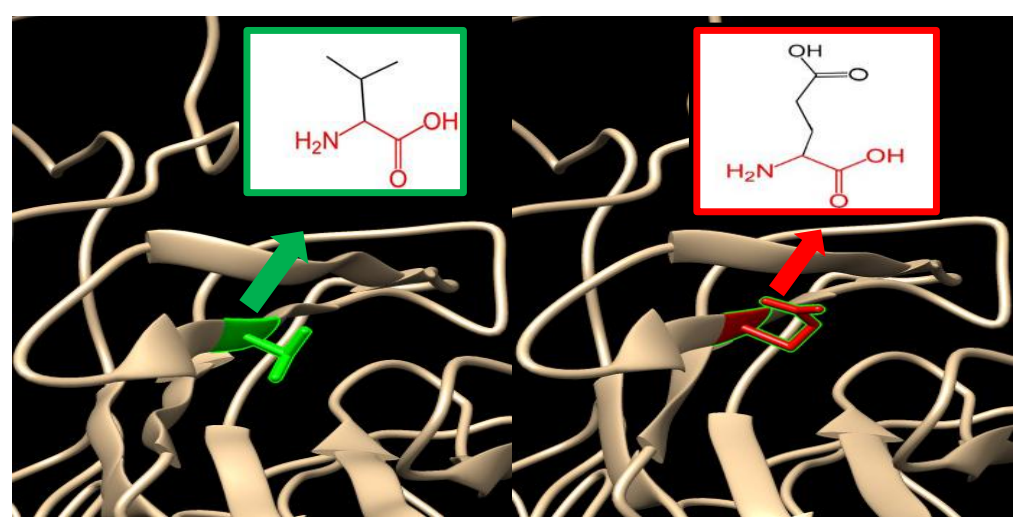

Figure 35: (rs1312638005): (V223E): The amino acid Valine change to Glutamic acid at position 223.

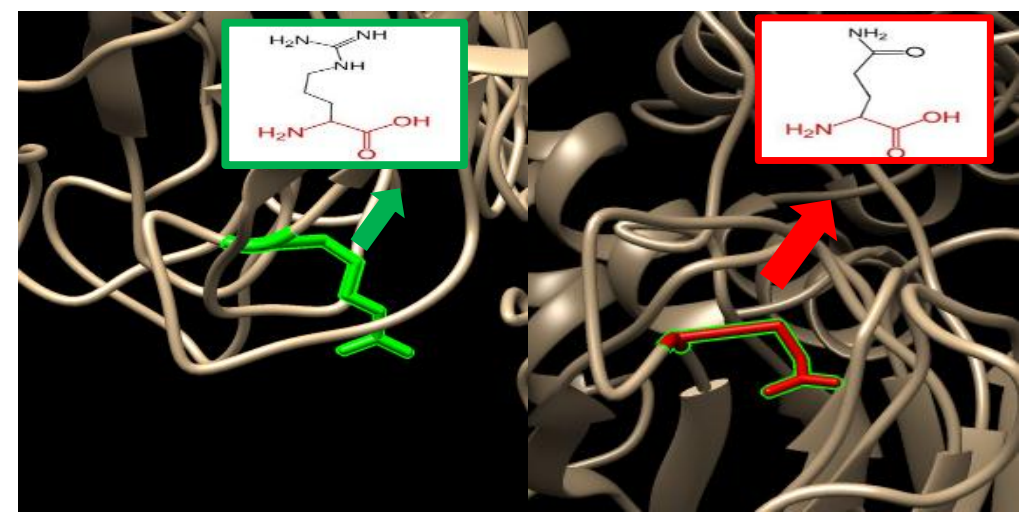

Figure 36: (rs749382362): (R219Q): The amino acid Arginine change to Glutamine at position 219.

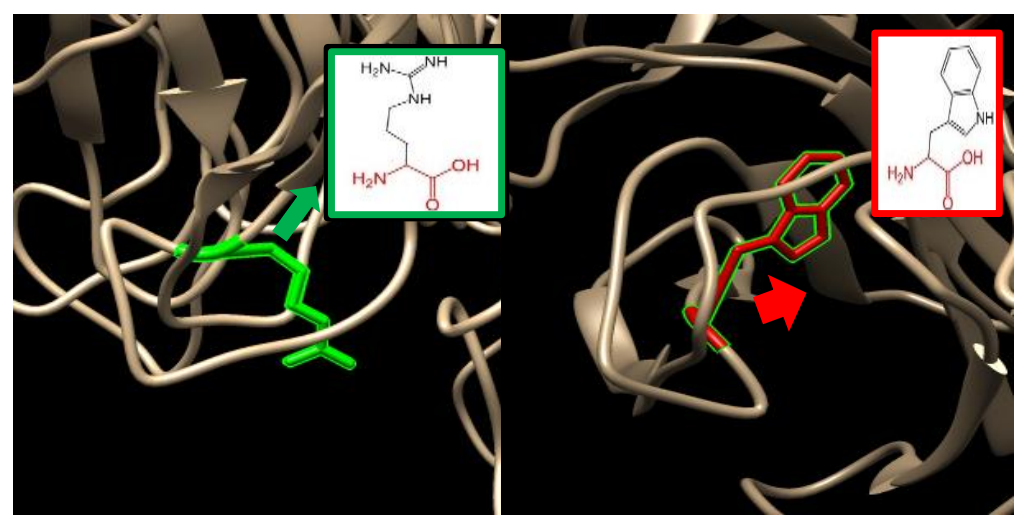

Figure 37: (rs374238430): (R219W): The amino acid Arginine changes to Tryptophan at position 219. 
bioRxiv preprint doi: https://doi.org/10.1101/436071; this version posted October 5, 2018. The copyright holder for this preprint (which was not certified by peer review) is the author/funder, who has granted bioRxiv a license to display the preprint in perpetuity. It is made available under

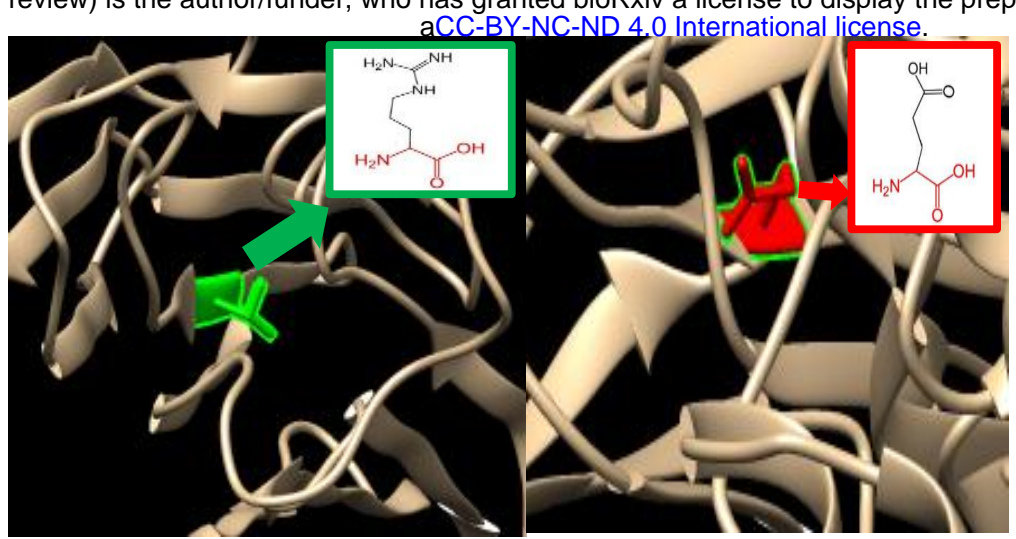

Figure 38: (rs1039834492): (V209E): The amino acid Valine change to Glutamic acid at position 209.

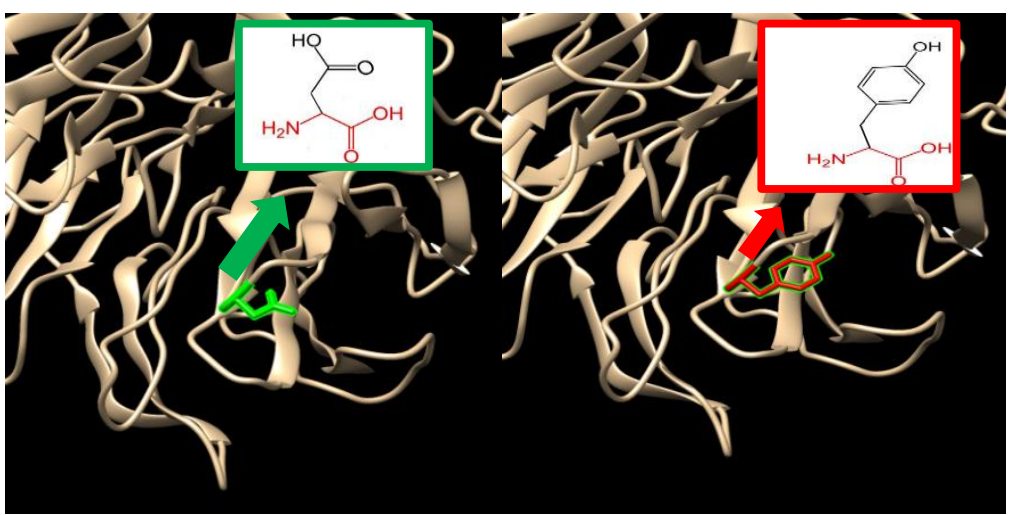

Figure 39: (rs747349523): (D203Y): The amino acid Aspartic acid change to tyrosine at position 203.

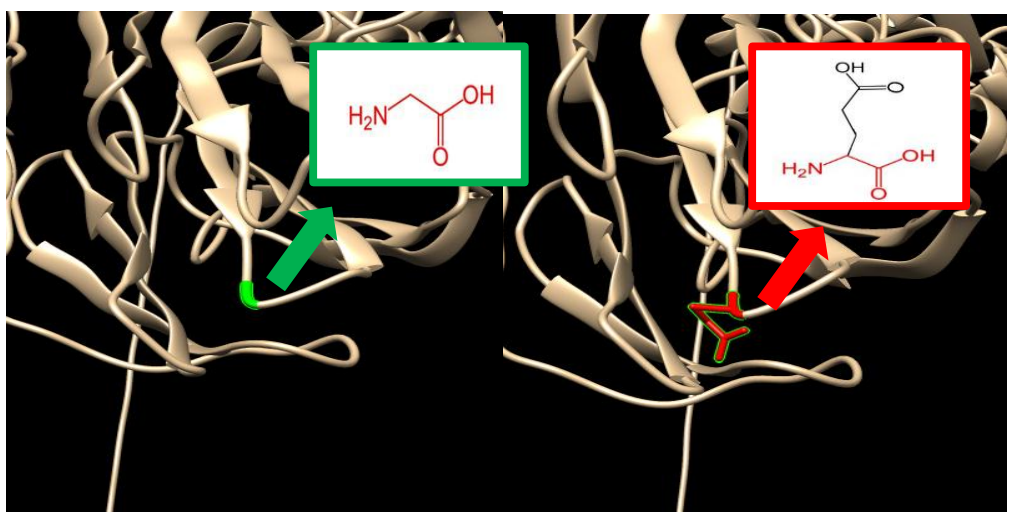

Figure 40: (rs776300430): (G202E): The amino acid Glycine change to Glutamic acid at position 202, Figure was done by chimera 1.8 . 
bioRxiv preprint doi: https://doi.org/10.1101/436071; this version posted October 5, 2018. The copyright holder for this preprint (which was not certified by peer review) is the author/funder, who has granted bioRxiv a license to display the preprint in perpetuity. It is made available under aCC-BY-NC-ND 4.0 International license.

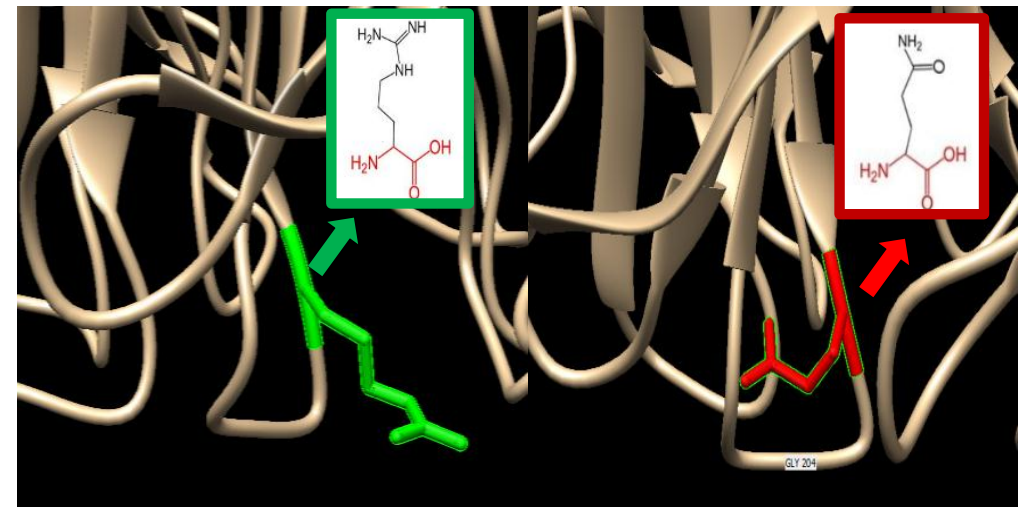

Figure 41: (rs367552387): (R201Q): The amino acid Arginine change to Glutamine at position 201.

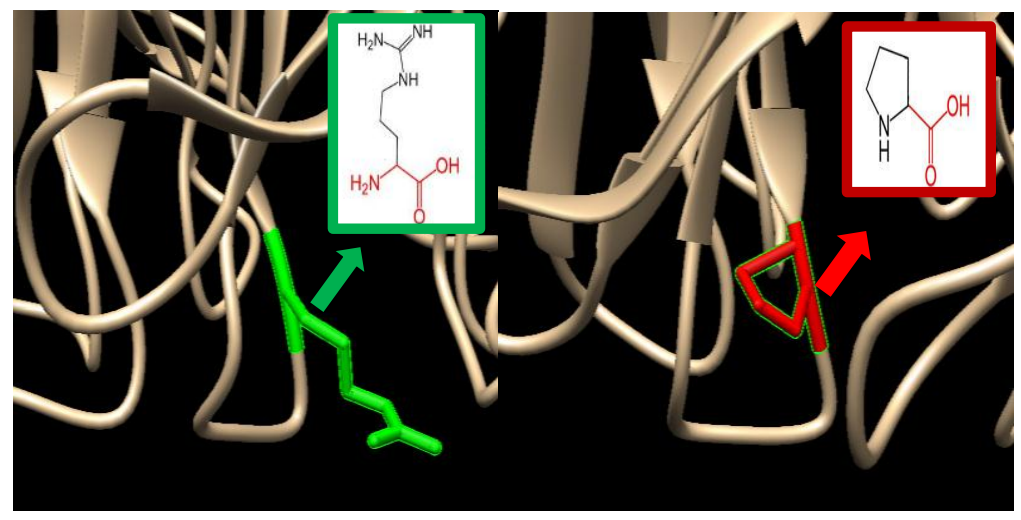

Figure 42: (R201P): The amino acid Arginine change to Proline at position 201.

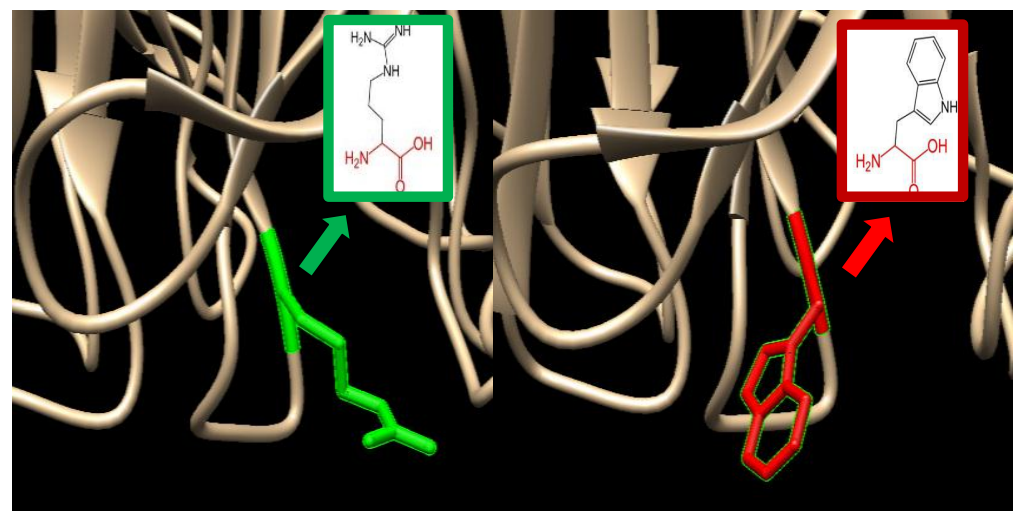

Figure 43: (rs773117166): (R201W): The amino acid Arginine change to Tryptophan at position 201. 


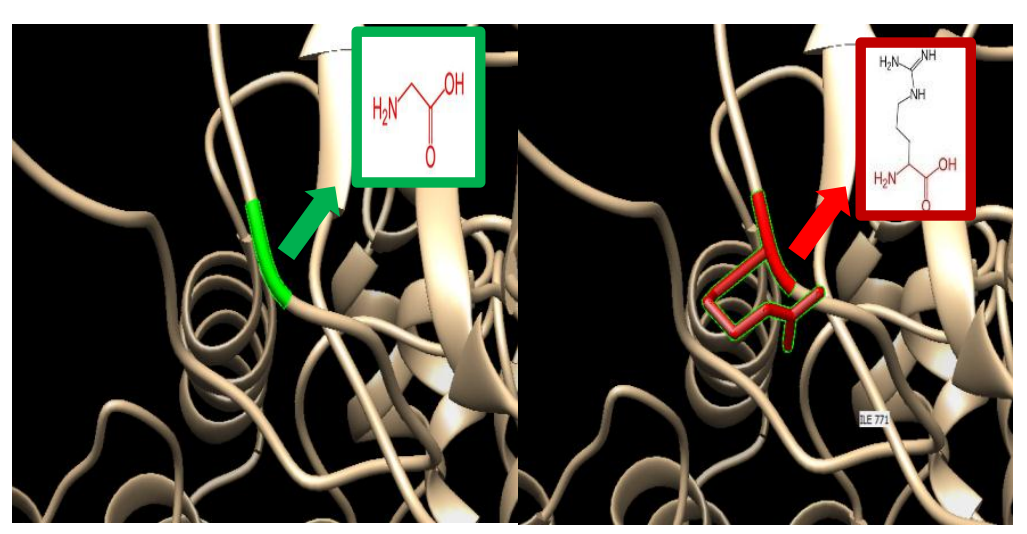

Figure 44: (rs565257577): (G162R): The amino acid Glycine change to Arginine at position 162.

\section{Discussion:}

We found 41 novel mutations (table3) that has effect on the stability and function of the IKBKAP gene using bioinformatics tools. The methods used are based on different aspects and parameters describing the pathogenicity and provide clues on the molecular level about the effect of mutations. It is not easy to predict the pathogenic effect of SNPs using single method. Therefore, we used multiple methods to compare and rely on the results predicted. In this study we used different in silico prediction algorithms: SIFT, PolyPhen-2, Provean, SNAP2, SNP\&GO, PHD-SNP, P-MUT and I-Mutant 3.0 ,Figure (2).

This study identified the total number of nSSNP in Homo sapiens located in coding region of IKBKAP gene, that were investigated in dbSNP/NCBI database ${ }^{(22)}$ out of 1458 there are 973 nsSNPs (missense mutations), which were submitted to SIFT server, PolyPhen-2 server, Provean sever and SNAP2 respectively, 291 (130 SNPs with Score 0) SNPs were predicted to be deleterious in SIFT server. In PolyPhen-2 server, our result showed that 570 were found to be damaging (159 possibly damaging and 411 probably damaging showed deleterious). In Provean server our result showed that 390 SNPs were predicted to be deleterious. While in SNAP2 server our result showed that 389 SNPs were predicted to be Effect. The differences in prediction capabilities refer to the fact that every prediction algorithm uses different sets of sequences and alignments. In table (2) we were submitted four positive results from SIFT, PolyPhen-2, Provean and SNAP2 to observe the disease causing one by SNP\&GO, PHD-SNP and P-Mut servers. Figures (1 \& 2).

In SNP\&GO, PHD-SNP and P-Mut software's were used to predict the association of SNPS with disease. According to SNP\&GO, PHD-SNP and P-Mut (69, 69 and 69 SNPs respectively) were found to be disease related SNPs. We selected the triple disease related SNPs only in 3 softwares for further analysis by I-Mutant 3.0 ,(Table 3). While I-Mutant result revealed that the 
protein stability decreased ( which destabilize the amino acid interaction) in the following SNPs: (R1137C, Y943N, 1936T, A907T, Y898C, D886V, R749K ,R722Q, L716S,Q710R, R693W ,R689Q ,R689W, G576D ,P410L ,G396V ,L336S ,H329Q ,Y328S, S298F, G272R, P248L, V223E, R219Q, R219W, V209E, G202E, R201P ,R201Q ,R201W ,G162R). While (Y992C, Y943C, N888Y, Y797C, T793R, D791V, Y328C, Y328F, W323R, D203Y) are found to increase the protein stability (Table 3). Figures (1\&2).

GeneMANIA revealed that IKBKAP gene has many important functions: DNA-directed RNA polymerase complex, DNA-directed RNA polymerase II, holoenzyme, DNA-templated transcription, elongation, nuclear DNA-directed RNA polymerase complex, positive regulation of cell migration, positive regulation of cell motility, positive regulation of locomotion, RNA polymerase complex, transcription elongation factor complex, transcription elongation from RNA polymerase II promoter. The genes co-expressed with, share similar protein domain, or participate to achieve similar function are illustrated by GeneMANIA and shown in figures ( 4 to 44) Table (4 \& 5).

We also used Project HOPE server to submit the 41 most deleterious (damaging) nsSNPs: (rs377679012):(R1137C):Cysteine (The mutant residue) is smaller than Arginine (the wild-type residue.) The wild-type residue charge was POSITIVE; the mutant residue charge is NEUTRAL. The mutant residue is more hydrophobic than the wild-type residue there is a difference in charge between the wild-type and mutant amino acid. The charge of the wild-type residue will be lost; this can cause loss of interactions with other molecules or residues. The wild-type and mutant amino acids differ in size. The mutant residue is smaller; this might lead to loss of interactions. The hydrophobicity of the wild-type and mutant residue differs .The mutation introduces a more hydrophobic residue at this position. This can result in loss of hydrogen bonds and/or disturb correct folding.

(rs372723869):(Y992C): Cysteine (the mutant residue) is smaller than the Tyrosine (wild-type residue). The mutant residue is more hydrophobic than the wild-type residue .The wild-type residue is very conserved, but a few other residue types have been observed at this position too. The mutant residue was not among the other residue types observed at this position in other, homologous proteins. The wild-type and mutant amino acids differ in size. The mutant residue is smaller than the wild-type residue. The mutation will cause an empty space in the core of the protein. The hydrophobicity of the wild-type and mutant residue differs . The mutation will cause loss of hydrogen bonds in the core of the protein and as a result disturb correct folding.

(rs924119518):(Y943C): Cysteine (the mutant residue) is smaller than Tyrosine (the wild-type residue) the mutant residue is more hydrophobic than the wild-type residue. The wild-type residue is very conserved, but a few other residue types have been observed at this position 
too. The mutant residue was not among the other residue types observed at this position in other, homologous proteins and is located near a highly conserved position. The wild-type and mutant amino acids differ in size. The mutant residue is smaller than the wild-type residue. This will cause a possible loss of external interactions .The hydrophobicity of the wild-type and mutant residue differs.

(rs1319436059):(Y943N): Asparagine (the mutant residue) is smaller than Tyrosine (the wildtype residue).The wild-type residue is more hydrophobic than the mutant residue and it is very conserved, but a few other residue types have been observed at this position too. The mutant residue was not among the other residue types observed at this position in other, homologous proteins and it is located near a highly conserved position. The wild-type and mutant amino acids differ in size. The mutant residue is smaller than the wild-type residue. This will cause a possible loss of external interactions. The hydrophobicity of the wild-type and mutant residue differs. The mutation might cause loss of hydrophobic interactions with other molecules on the surface of the protein.

(rs199617267):(1936T):Threonine(The mutant residue) is smaller than the Isoleucine (wild-type residue).The wild-type residue is more hydrophobic than the mutant residue. The wild-type and mutant amino acids differ in size. The mutant residue is smaller than the wild-type residue. The mutation will cause an empty space in the core of the protein. The mutation will cause loss of hydrophobic interactions in the core of the protein.

(rs1218321803):(A907T): Threonine (The mutant residue) is bigger than Alanine (the wild-type residue). The wild-type residue is more hydrophobic than the mutant residue. The mutation converts the wild-type residue in a residue that does not prefer $\alpha$-helices as secondary structure. The wild-type residue is very conserved, but a few other residue types have been observed at this position too. The wild-type and mutant amino acids differ in size. The mutant residue is bigger than the wild-type residue. The wild-type residue was buried in the core of the protein. The mutant residue is bigger and probably will not fit. The hydrophobicity of the wildtype and mutant residue differs. The mutation will cause loss of hydrophobic interactions in the core of the protein.

(rs199679232):(Y898C): Cysteine (The mutant residue) is smaller than (the wild-type residue). The mutant residue is more hydrophobic than the wild-type residue. The size difference between wild-type and mutant residue makes that the new residue is not in the correct position to make the same hydrogen bond as the original wild-type residue did. The difference in hydrophobicity will affect hydrogen bond formation. The wild-type residue is very conserved, but a few other residue types have been observed at this position too. The wild-type and mutant amino acids differ in size. The mutant residue is smaller than the wild-type residue. This will cause a possible loss of external interactions. The hydrophobicity of the wild-type and mutant residue differs. 
(rs752349406):(N888Y): Tyrosine (The mutant residue) is bigger than (the wild-type residue). The mutant residue is more hydrophobic than the wild-type residue. The wild-type residue is very conserved, but a few other residue types have been observed at this position too. The mutant residue was not among the other residue types observed at this position in other, homologous proteins. However, residues that have some properties in common with your mutated residue were observed. This means that in some rare cases the mutation might occur without damaging the protein. The mutated residue is located on the surface of a domain with unknown function. The wild-type and mutant amino acids differ in size. The mutant residue is bigger than the wild-type residue. The residue is located on the surface of the protein; mutation of this residue can disturb interactions with other molecules or other parts of the protein. The hydrophobicity of the wild-type and mutant residue differs.

(rs1270126477);( D886V): Valine (The mutant residue) is smaller than Aspartic Acid (the wildtype residue). The wild-type residue charge was NEGATIVE; the mutant residue charge is NEUTRAL. The mutant residue is more hydrophobic than the wild-type residue. The size difference between wild-type and mutant residue makes that the new residue is not in the correct position to make the same hydrogen bond as the original wild-type residue did. The difference in hydrophobicity will affect hydrogen bond formation. The mutated residue is located on the surface of a domain with unknown function. There is a difference in charge between the wild-type and mutant amino acid. The charge of the wild-type residue is lost by this mutation. This can cause loss of interactions with other molecules. The wild-type and mutant amino acids differ in size. The mutant residue is smaller than the wild-type residue. This will cause a possible loss of external interactions. The hydrophobicity of the wild-type and mutant residue differs.

(rs1181862976):(Y797C): cysteine (The mutant residue) is smaller than tyrosine (the wild-type residue). Each amino acid has it is own specific size charge, and hydrophobicity-value. The original wild-type residue and newly introduced mutant residue often differ in the properties .The mutant residue is smaller than the wild-type residue. The mutant residue is more hydrophobic than the wild-type residue.

(rs201596987):(T793R): Arginine (The mutant residue) is bigger than Threonine ( The wile-type residue). The wild-type residue is very conserved, but a few other residue types have been observed at this position too. Is buried in the core of a domain. The differences between the wild-type and mutant residue might disturb the core structure of this domain. There is a difference in charge between the wild-type and mutant amino acid. The mutant residue introduces a charge in a buried residue which can lead to protein folding problems. The wildtype and mutant amino acids differ in size. The mutant residue is bigger than wild-type residue. 
The wild-type residue was buried in the core of the protein. The mutant residue is bigger and probably will not fit. The hydrophobicity of the wild-type and mutant residue differ. The mutation will cause loss of hydrophobic interaction in the core of the protein.

(D791V): Valine (The mutant residue) is smaller than Aspartic Acid (The wild-type residue). The wild-type residue is very conserved, but a few other residue types have been observed at this position too. The mutant residue was not among the other residue types observed at this position in other, homologous proteins. However, the residues that have in some rare cases the mutation might occur without damaging the protein. The mutant residue is located on the surface of a domain with unknown function. The residue was not found to be in contact with other domains of which the function is known without the used structure. There is a difference in charge between the wild-type and mutant amino acid. The wild-type and mutant amino acids differ in size. The mutant reside is smaller than wild-type residue. This will cause a possible loss of external interactions. The hydrophobicity of the wild-type and mutant reside differs.

(rs1452809051):(R749K): Lysine (The mutant residue) is smaller than Arginine ( wild-type residue). The wild-type residue is very conserved, but a few other residue types have been observed at this position too. The mutant residue was not among the other residue types observed at this position in other, homologous proteins. Only this type was found at this position. Mutation of a $100 \%$ conserved residue is usually damaging for the protein. This mutation might occur in some rare cases, but it is more likely that the mutation is damaging to the protein. The mutated residue is located on the surface of a domain with unknown function. The residue was not found to be in contact with other domains of which the function is known within the used structure. However, contact with other molecules or domains is still possible and might be affected this mutation. The wild-type and mutant amino acid differ in size. The mutant residue is smaller than the wild-type residue. This will cause a possible loss of external interactions.

(rs370575901):(R722Q): Glutamine ( The mutant residue) is smaller than Arginine ( wild-type residue ). The wild-type residue charge was POSITIVE. The mutant residue charge is NATURAL. Only this residue type was found at this position. Mutation of a $100 \%$ conserved residue is usually damaging for the protein. This mutation might occur in some rare cases, but it is more likely that the mutation is damaging to the protein. The mutant residue is located near a highly conserved position. The mutated residue is located on the surface of a domain with unknown function. The residue was not found to be in contact with other domains of which the function is known within the used structure. There is a difference charge between the wild-type and mutant amino acid. The charge of the wild-type residue is lost by this mutation. This can cause loss of interactions. The wild-type and mutant amino acids differ in size. The mutant residue is smaller than the wild-type residue. This will cause a possible loss of external interactions. 
(rs767003999): (L716S): Serine (The mutant residue) is smaller than Leucine (The wild-type residue). The mutation introduces an amino acid with different properties, which can disrupt this domain and abolish its function. The mutant residue is less hydrophobic than the wild-type residue. Hydrophobic interactions, either in the core of the protein or on the surface, will be lost.

(rs1275065654): (Q710R): Arginine (The mutant residue) is bigger than Glutamine (The wildtype residue). The increases in the size of the mutant residue might lead to bumps. The mutant residue is positively charge, while the wild-type residue charge is neutral. This charge changes could lead to repulsion of ligands or other residues with the same charge.

(rs763692410): (R693W): Tryptophan (The mutant residue) is bigger than Arginine (The wildtype residue). The increases in the size of the mutant residue might lead to bumps. The mutant residue charge is neutral, while the wild-type residue is positively charged. This change can cause loss of interactions with other molecules or residues. The mutant residue is more hydrophobic than the wild-type residue. This can result in loss of hydrogen bonds and/or disturb correct folding.

(rs201742754): (R689Q): Glutamine (The mutant residue) is smaller than Arginine (The wildtype residue). This might lead to loss of interactions. The mutant residue is neutral in charge, while the wild-type residue is positively charged. The loss of this charge can cause loss of interactions with other molecules or residues.

(rs201390288): (R689W): Tryptophan (The mutant residue) is bigger than Arginine (The wildtype residue).The increases in the size of the mutant residue might lead to bumps. The mutant residue charge is neutral, while the wild-type residue is positively charged. This change can cause loss of interactions with other molecules or residues. The mutant residue is more hydrophobic than the wild-type residue. This can result in loss of hydrogen bonds and/or disturb correct folding.

(rs56229130):(G576D): Aspartic Acid (the mutant residue) is bigger than glycine (the wild type).The wild-type residue charge was neutral, the mutant residue charge is negative. The wild-type residue is more hydrophobic than the mutant residue. The wild type residue is the most flexible of all residues. This flexibility might be necessary for the protein's function. Mutation of this glycine can abolish this function. There is a difference in charge between the wild type and mutant amino acid. The mutation introduces a charge; this can cause repulsion of ligands or other residues with the same charge. The wild type and mutant amino acids differ in size. The mutant residue is bigger, this might lead to bumps. The torsion angles for this residue are unusual. Only glycine is flexible enough to make these torsion angles, mutation into another 
residue will force the local backbone into an incorrect conformation and will disturb the local structure.

(rs375957332):(P410L): Leucine (The mutant residue) is bigger than the Proline (The wild type). Proline are known to be very rigid and therefore induce a special backbone conformation, which might be required at this position. The mutation can disturb this special conformation, there by disturbing the local structure. The wild type and mutant amino acids differ in size. The mutant residue is bigger this might lead to bumps.

(rs1414061926):(G396V): Valine (the mutant residue) is bigger than Glycine (the wild type)the mutant residue is more hydrophobic than the wild-type residue. The torsion angles for this residue are unusual. Only glycine is flexible enough to make these torsion angles, mutation into another residue will force the local backbone into an incorrect conformation and will disturb the local structure. The wild type and mutant amino acids differ in size. The mutant residue is bigger this might lead to bumps.

(rs775321513):(L336S): Serine (the mutant residue) is smaller than leucine (the wild type residue). The wild type residue is more hydrophobic than the mutant residue. The hydrophobicity of the wild type and mutant residue differs, so hydrophobic interactions, either in the core of the protein or on the surface, will be lost. The wild type and mutant amino acids differ in size. The mutant residue is smaller; this might lead to loss of interactions.

(rs764033545):(H329Q): Histidine (the mutant residue) is smaller than glutamine (the wild type), the wild-type and mutant amino acids differ in size. The mutant residue is smaller; this might lead to loss of interactions.

(rs750663365):(Y328S): Serine (The mutant residue) is smaller than Tyrosine (the wild-type residue).The wild-type residue is predicted (using the Reprof software) to be located in its preferred secondary structure, a $\beta$-strand. The mutant residue prefers to be in another secondary structure; therefore the local conformation will be slightly destabilized. The wildtype and mutant amino acids differ in size. The mutant residue is smaller; this might lead to loss of interactions.

(Y328C): cysteine (The mutant residue) is smaller than (the wild-type residue). The mutant residue is more hydrophobic than the wild-type residue. The wild-type residue is predicted (using the Reprof software) to be located in its preferred secondary structure, a $\beta$-strand. The mutant residue prefers to be in another secondary structure; therefore the local conformation will be slightly destabilized. The wild-type and mutant amino acids differ in size. The mutant residue is smaller; this might lead to loss of interactions. The hydrophobicity of the wild-type and mutant residue differs. The mutation introduces a more hydrophobic residue at this position. This can result in loss of hydrogen bonds and/or disturb correct folding. 
(Y328F): Phenylalanine (The mutant residue) is bigger than Tyrosine (the wild-type residue).The mutant residue is more hydrophobic than the wild-type residue. The wild-type and mutant amino acids differ in size. The mutant residue is smaller; this might lead to loss of interactions. The hydrophobicity of the wild-type and mutant residue differs. The mutation introduces a more hydrophobic residue at this position. This can result in loss of hydrogen bonds and/or disturb correct folding.

(rs1159235595):(W323R): Arginine (The mutant residue) is smaller than Tryptophan (the wildtype residue).The wild-type residue charge was NEUTRAL, the mutant residue charge is POSITIVE. The wild-type residue is predicted (using the Reprof software) to be located in its preferred secondary structure, a $\beta$-strand. The mutant residue prefers to be in another secondary structure; therefore the local conformation will be slightly destabilized. The wildtype residue is more hydrophobic than the mutant residue. The wild-type and mutant amino acids differ in size. The mutant residue is smaller; this might lead to loss of interactions. The hydrophobicity of the wild-type and mutant residue differs. Hydrophobic interactions, either in the core of the protein or on the surface, will be lost.

(rs1288706972):(S298F): Phenylalanine (The mutant residue) is bigger than Serine (the wildtype residue).The mutant residue is more hydrophobic than the wild-type residue. The wildtype residue is predicted (using the Reprof software) to be located in its preferred secondary structure, a turn. The mutant residue prefers to be in another secondary structure; therefore the local conformation will be slightly destabilized. The wild-type and mutant amino acids differ in size. The mutant residue is bigger, this might lead to bumps. The hydrophobicity of the wildtype and mutant residue differs. The mutation introduces a more hydrophobic residue at this position. This can result in loss of hydrogen bonds and/or disturb correct folding.

()(G272R) Arginine (The mutant residue) is bigger than Glycine (the wild-type residue). The wildtype residue charge was NEUTRAL, the mutant residue charge is POSITIVE. The wild-type residue is more hydrophobic than the mutant residue. There is a difference in charge between the wild-type and mutant amino acid. The mutation introduces a charge, this can cause repulsion of ligands or other residues with the same charge. The wild-type and mutant amino acids differ in size. The mutant residue is bigger, this might lead to bumps. The torsion angles for this residue are unusual. only glycine is flexible enough to make these torsion angles, mutation into another residue will force the local backbone into an incorrect conformation and will disturb the local structure.

(rs1394468770):(P248L): Leucine (The mutant residue) is bigger than Proline (The wild-type residue). The mutated residue is located in a domain that is important for binding of other molecules. Mutation of the residue might disturb this function. The wild-type and mutant amino acids differ in size. The mutant residue is bigger, this might lead to bumps. Prolines are 
known to have a very rigid structure, sometimes forcing the backbone in a specific conformation. Possibly, the mutation changes a proline with such a function into another residue, thereby disturbing the local structure.

(rs1312638005):(V223E): Glutamic acid (The mutant residue) is bigger than Valine (The wildtype residue). The wild-type residue charge was NEUTRAL; the mutant residue charge is NEGATIVE. The wild-type residue is more hydrophobic than the mutant residue. There is a difference in charge between the wild-type and mutant amino acid. The mutation introduces a charge, this can cause repulsion of ligands or other residues with the same charge. The wildtype and mutant amino acids differ in size. The mutant residue is bigger, this might lead to bumps. The hydrophobicity of the wild-type and mutant residue differs. Hydrophobic interactions, either in the core of the protein or on the surface, will be lost.

(rs749382362):(R219Q): Glutamine (The mutant residue) is smaller than Arginine (The wild-type residue). The wild-type residue charge was POSITIVE; the mutant residue charge is NEUTRAL. The mutated residue is located in a domain that is important for binding of other molecules. Mutation of the residue might disturb this function. There is a difference in charge between the wild-type and mutant amino acid. The charge of the wild-type residue will be lost; this can cause loss of interactions with other molecules or residues. The wild-type and mutant amino acids differ in size. The mutant residue is smaller; this might lead to loss of interactions.

(rs374238430):(R219W): Tryptophan (The mutant residue) is bigger than Arginine (The wildtype residue). The wild-type residue charge was POSITIVE; the mutant residue charge is NEUTRAL. The mutant residue is more hydrophobic than the wild-type residue. The mutated residue is located in a domain that is important for binding of other molecules. Mutation of the residue might disturb this function. There is a difference in charge between the wild-type and mutant amino acid. The charge of the wild-type residue will be lost; this can cause loss of interactions with other molecules or residues. The wild-type and mutant amino acids differ in size. The mutant residue is bigger, this might lead to bumps. The hydrophobicity of the wildtype and mutant residue differs. The mutation introduces a more hydrophobic residue at this position. This can result in loss of hydrogen bonds and/or disturb correct folding.

(rs1039834492):(V209E): Glutamic Acid (The mutant residue) is bigger than Valine (The wildtype residue). The wild-type residue charge was NEUTRAL; the mutant residue charge is NEGATIVE. The wild-type residue is more hydrophobic than the mutant residue. The mutated residue is located in a domain that is important for binding of other molecules. Mutation of the residue might disturb this function. There is a difference in charge between the wild-type and mutant amino acid. The mutation introduces a charge; this can cause repulsion of ligands or other residues with the same charge. The wild-type and mutant amino acids differ in size. The mutant residue is bigger, this might lead to bumps. The hydrophobicity of the wild-type and mutant residue differs. Hydrophobic interactions, either in the core of the protein or on the surface, will be lost. 
(rs747349523):(D203Y): Tyrosine (The mutant residue) is bigger than Aspartic Acid ( the wildtype residue).The wild-type residue charge was NEGATIVE, the mutant residue charge is NEUTRAL .The mutant residue is more hydrophobic than the wild-type residue There is a difference in charge between the wild-type and mutant amino acid. The charge of the wild-type residue will be lost, this can cause loss of interactions with other molecules or residues. The wild-type and mutant amino acids differ in size. The mutant residue is bigger, this might lead to bumps. The hydrophobicity of the wild-type and mutant residue differs .The mutation introduces a more hydrophobic residue at this position. This can result in loss of hydrogen bonds and/or disturb correct folding.

(rs776300430):(G202E): Glutamic Acid (The mutant residue) is bigger than Glycine( the wildtype residue). The wild-type residue charge was NEUTRAL, the mutant residue charge is NEGATIVE.The wild-type residue is more hydrophobic than the mutant residue there is a difference in charge between the wild-type and mutant amino acid. The mutation introduces a charge; this can cause repulsion of ligands or other residues with the same charge. The wildtype and mutant amino acids differ in size. The mutant residue is bigger, this might lead to bump. The torsion angles for this residue are unusual. Only glycine is flexible enough to make these torsion angles, mutation into another residue will force the local backbone into an incorrect conformation and will disturb the local structure.

(rs367552387):(R201Q): Glutamine (The mutant residue) is smaller than Arginine (the wild-type residue).The wild-type residue charge was POSITIVE, the mutant residue charge is NEUTRAL. There is a difference in charge between the wild-type and mutant amino acid. The charge of the wild-type residue will be lost, this can cause loss of interactions with other molecules or residues. The wild-type and mutant amino acids differ in size .The mutant residue is smaller, this might lead to loss of interactions.

()(R201P): Proline (The mutant residue) is smaller than Arginine (the wild-type residue).The wild-type residue charge was POSITIVE, the mutant residue charge is NEUTRAL.The mutant residue is more hydrophobic than the wild-type residue There is a difference in charge between the wild-type and mutant amino acid. The charge of the wild-type residue will be lost, this can cause loss of interactions with other molecules or residues. The wild-type and mutant amino acids differ in size. The mutant residue is smaller, this might lead to loss of interactions. The hydrophobicity of the wild-type and mutant residue differs. The mutation introduces a more hydrophobic residue at this position. This can result in loss of hydrogen bonds and/or disturb correct folding.

(rs773117166)(R201W) Tryptophan (The mutant residue) is bigger than Arginine (the wild-type residue.)The wild-type residue charge was POSITIVE, the mutant residue charge is NEUTRAL The mutant residue is more hydrophobic than the wild-type residue There is a difference in charge 
between the wild-type and mutant amino acid. The charge of the wild-type residue will be lost, this can cause loss of interactions with other molecules or residues. The wild-type and mutant amino acids differ in size. The mutant residue is bigger, this might lead to bumps. The hydrophobicity of the wild-type and mutant residue differs. The mutation introduces a more hydrophobic residue at this position. This can result in loss of hydrogen bonds and/or disturb correct folding.

(rs565257577)(G162R) Arginine (The mutant residue) is bigger than Glycine( the wild-type residue).The wild-type residue charge was NEUTRAL, the mutant residue charge is POSITIVE. The wild-type residue is more hydrophobic than the mutant residue. There is a difference in charge between the wild-type and mutant amino acid. The mutation introduces a charge, this can cause repulsion of ligands or other residues with the same charge. The wild-type and mutant amino acids differ in size. The mutant residue is bigger, this might lead to bumps. The torsion angles for this residue are unusual. only glycine is flexible enough to make these torsion angles, mutation into another residue will force the local backbone into an incorrect conformation and will disturb the local structure.

In the light of this study, any one with relation to Jewish ancestors or suspected of being a half Jewish, should be consider to do Exome sequencing for IKBKAP gene to check Familial dysautonomia disease.

\section{Conclusion:}

In the current work the influence of functional SNPs in the IKBKAP gene was investigated through various computational methods, and determined that (R1137C, Y992C, Y943C, Y943N, 1936T, A907T, Y898C, N888Y, D886V, Y797C, T793R, D791V, R749K, R722Q, L716S, Q710R, R693W, R689Q, R689W, G576D, P410L, G396V, L336S, H329Q, Y328S, Y328C, Y328F, W323R, S298F, G272R, P248L, V223E, R219Q, R219W, V209E, D203Y, G202E, R201Q, R201P, R201W, G162R) new SNPs have a potential functional impact and can thus be used as diagnostic markers.

\section{Acknowledgment:}

The authors wish to express their profound gratitude and deep regards to Africa City of Technology - Sudan.

\section{Conflict of interest:}

The authors declare that they have no competing interests.

\section{Data availability statement:}


Data available on request from the authors, the data that support the findings of this study are available from the cross ponding author upon reasonable request.

\section{References:}

1. Axelrod FB. Familial dysautonomia. Muscle \& nerve. 2004;29(3):352-63.

2. Axelrod FB, Hilz MJ. Inherited autonomic neuropathies. Seminars in neurology. 2003;23(4):381-

90.

3. Donadon I, Pinotti M, Rajkowska K, Pianigiani G, Barbon E, Morini E, et al. Exon-specific U1 snRNAs improve ELP1 exon 20 definition and rescue ELP1 protein expression in a familial dysautonomia mouse model. Human molecular genetics. 2018;27(14):2466-76.

4. Palma JA, Norcliffe-Kaufmann L, Fuente-Mora C, Percival L, Mendoza-Santiesteban C, Kaufmann H. Current treatments in familial dysautonomia. Expert opinion on pharmacotherapy. 2014;15(18):265371.

5. Slaugenhaupt SA, Blumenfeld A, Gill SP, Leyne M, Mull J, Cuajungco MP, et al. Tissue-specific expression of a splicing mutation in the IKBKAP gene causes familial dysautonomia. American journal of human genetics. 2001;68(3):598-605.

6. Carroll MS, Kenny AS, Patwari PP, Ramirez JM, Weese-Mayer DE. Respiratory and cardiovascular indicators of autonomic nervous system dysregulation in familial dysautonomia. Pediatric pulmonology. 2012;47(7):682-91.

7. Palma JA, Norcliffe-Kaufmann L, Perez MA, Spalink CL, Kaufmann H. Sudden Unexpected Death During Sleep in Familial Dysautonomia: A Case-Control Study. Sleep. 2017;40(8).

8. Dietrich P, Dragatsis I. Familial Dysautonomia: Mechanisms and Models. Genetics and molecular biology. 2016;39(4):497-514.

9. Dietrich P, Alli S, Shanmugasundaram R, Dragatsis I. IKAP expression levels modulate disease severity in a mouse model of familial dysautonomia. Human molecular genetics. 2012;21(23):5078-90.

10. Di Santo R, Bandau S, Stark MJ. A conserved and essential basic region mediates tRNA binding to the Elp1 subunit of the Saccharomyces cerevisiae Elongator complex. Molecular microbiology. 2014;92(6):1227-42.

11. Xu H, Lin Z, Li F, Diao W, Dong C, Zhou H, et al. Dimerization of elongator protein 1 is essential for Elongator complex assembly. Proceedings of the National Academy of Sciences of the United States of America. 2015;112(34):10697-702.

12. Lehavi O, Aizenstein O, Bercovich D, Pavzner D, Shomrat R, Orr-Urtreger A, et al. Screening for familial dysautonomia in Israel: evidence for higher carrier rate among Polish Ashkenazi Jews. Genetic testing. 2003;7(2):139-42.

13. Sinha R, Kim YJ, Nomakuchi T, Sahashi K, Hua Y, Rigo F, et al. Antisense oligonucleotides correct the familial dysautonomia splicing defect in IKBKAP transgenic mice. Nucleic acids research. 2018;46(10):4833-44.

14. Salani M, Urbina F, Brenner A, Morini E, Shetty R, Gallagher CS, et al. Development of a Screening Platform to Identify Small Molecules That Modify ELP1 Pre-mRNA Splicing in Familial Dysautonomia. SLAS discovery : advancing life sciences R \& D. 2018:2472555218792264.

15. Leyne M, Mull J, Gill SP, Cuajungco MP, Oddoux C, Blumenfeld A, et al. Identification of the first non-Jewish mutation in familial Dysautonomia. American journal of medical genetics Part $A$.

2003;118A(4):305-8. 
16. Cuajungco MP, Leyne M, Mull J, Gill SP, Lu W, Zagzag D, et al. Tissue-specific reduction in splicing efficiency of IKBKAP due to the major mutation associated with familial dysautonomia. American journal of human genetics. 2003;72(3):749-58.

17. Kazachkov M, Palma JA, Norcliffe-Kaufmann L, Bar-Aluma BE, Spalink CL, Barnes EP, et al. Respiratory care in familial dysautonomia: Systematic review and expert consensus recommendations. Respiratory medicine. 2018;141:37-46.

18. Norcliffe-Kaufmann L, Axelrod FB, Kaufmann H. Developmental abnormalities, blood pressure variability and renal disease in Riley Day syndrome. Journal of human hypertension. 2013;27(1):51-5.

19. Mendoza-Santiesteban CE, Hedges lii TR, Norcliffe-Kaufmann L, Axelrod F, Kaufmann H. Selective retinal ganglion cell loss in familial dysautonomia. Journal of neurology. 2014;261(4):702-9.

20. Portnoy S, Maayan C, Tsenter J, Ofran Y, Goldman V, Hiller N, et al. Characteristics of ataxic gait in familial dysautonomia patients. PloS one. 2018;13(4):e0196599.

21. Bajard A, Chabaud S, Cornu C, Castellan AC, Malik S, Kurbatova P, et al. An in silico approach helped to identify the best experimental design, population, and outcome for future randomized clinical trials. Journal of clinical epidemiology. 2016;69:125-36.

22. Benson DA, Cavanaugh M, Clark K, Karsch-Mizrachi I, Lipman DJ, Ostell J, et al. GenBank. Nucleic acids research. 2017;45(D1):D37-d42.

23. Artimo P, Jonnalagedda M, Arnold K, Baratin D, Csardi G, de Castro E, et al. ExPASy: SIB bioinformatics resource portal. Nucleic acids research. 2012;40(Web Server issue):W597-603.

24. Sim NL, Kumar P, Hu J, Henikoff S, Schneider G, Ng PC. SIFT web server: predicting effects of amino acid substitutions on proteins. Nucleic acids research. 2012;40(Web Server issue):W452-7.

25. Adzhubei IA, Schmidt S, Peshkin L, Ramensky VE, Gerasimova A, Bork P, et al. A method and server for predicting damaging missense mutations. Nature methods. 2010;7(4):248-9.

26. Choi Y, Sims GE, Murphy S, Miller JR, Chan AP. Predicting the functional effect of amino acid substitutions and indels. PloS one. 2012;7(10):e46688.

27. Hecht $M$, Bromberg $Y$, Rost $B$. Better prediction of functional effects for sequence variants. BMC genomics. 2015;16 Suppl 8:S1.

28. Calabrese R, Capriotti E, Fariselli P, Martelli PL, Casadio R. Functional annotations improve the predictive score of human disease-related mutations in proteins. Human mutation. 2009;30(8):1237-44.

29. Lopez-Ferrando V, Gazzo A, de la Cruz X, Orozco M, Gelpi JL. PMut: a web-based tool for the annotation of pathological variants on proteins, 2017 update. Nucleic acids research.

2017;45(W1):W222-w8.

30. Capriotti E, Fariselli P, Casadio R. I-Mutant2.0: predicting stability changes upon mutation from the protein sequence or structure. Nucleic acids research. 2005;33(Web Server issue):W306-10.

31. Warde-Farley D, Donaldson SL, Comes O, Zuberi K, Badrawi R, Chao P, et al. The GeneMANIA prediction server: biological network integration for gene prioritization and predicting gene function. Nucleic acids research. 2010;38(Web Server issue):W214-20.

32. Wang S, Li W, Liu S, Xu J. RaptorX-Property: a web server for protein structure property prediction. Nucleic acids research. 2016;44(W1):W430-5. 